TITLE:

\title{
Exciton and Charge Dynamics in Polymer Solar Cells Studied by Transient Absorption Spectroscopy
}

$\operatorname{AUTHOR}(\mathrm{S})$ :

Ohkita, Hideo; Ito, Shinzaburo

\section{CITATION:}

Ohkita, Hideo ... [et al]. Exciton and Charge Dynamics in Polymer Solar Cells Studied by Transient Absorption Spectroscopy. Green Energy and Technology 2012: 103-137

ISSUE DATE:

2012-11

URL:

http://hdl.handle.net/2433/193054

\section{RIGHT:}

The final publication is available at Springer via http://dx.doi.org/10.1007/978-1-44714823-4_5.; This is not the published version. Please cite only the published version.; この 論文は出版社版でありません。引用の際には出版社版をご確認ご利用ください。 


\section{Exciton and Charge Dynamics in Polymer Solar Cells Studied by Transient Absorption Spectroscopy}

Hideo Ohkita, ${ }^{\mathrm{a}, \mathrm{b}, *}$ Shinzaburo Ito $^{\mathrm{a}}$

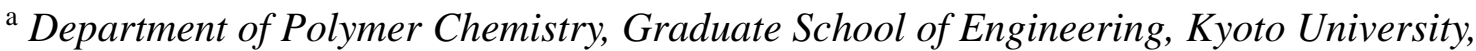
Katsura, Nishikyo, Kyoto 615-8510, Japan

b PRESTO, Japan Science and Technology Agency (JST),4-1-8, Honcho Kawaguchi, Saitama 332-0012, Japan

E-mail: ohkita@photo.polym.kyoto-u.ac.jp

FAX: +81-75-383-2617

TEL: +81-75-383-2613 


\section{Contents}

1. Introduction

2. Temporal Scale of Photovoltaic Conversion

3. Transient Absorption Spectroscopy

4. Assignment of Photoexcitations

4.1 Singlet and Triplet Excitons

4.2 Polarons

4.3 Other Charge Carriers

5. Exciton Dynamics

5.1 Exciton Delocalization

5.2 Energy Transfer

5.3 Triplet Formation

6. Charge Dynamics

\subsection{Charge Generation}

6.2 Geminate and Bimolecular Recombination

6.3 Trap-Free and Trap Limited Recombination

6.4 Relevance to Device Performance

7. Concluding Remarks

Acknowledgments

References 


\section{Introduction}

In recent years, polymer solar cells are attracting increasing interest because of their potential advantages: lightweight, flexibility, and high-throughput and large-area production based on the printing and coating techniques [1-3]. Although the power conversion efficiency (PCE) of polymer solar cells was less than 1\% in the 1990s, it has steadily increased every year in the past decade owing to extensive research. In particular, regioregular poly(3-hexylthiophene) (RR-P3HT) has been studied thoroughly as a donor material in polymer/fullerene solar cells [4-9]. It is noteworthy that this polymer has a good balance between solubility and optoelectronic properties [10-13], while most conjugate polymers generally have faced a trade-off between them. Regioregular P3HT is likely to be crystalline in solid films even with high solubility in various organic solvents because each monomer unit attached with a hexyl group is regularly bound in a head-to-tail linkage. Consequently, RR-P3HT:PCBM solar cells have been reported to be strongly dependent on the fabrication conditions, particularly annealing treatments. After thermal or solvent annealing, RR-P3HT:PCBM solar cells exhibit a reproducible PCE approaching 5\% and excellent external quantum efficiency (EQE) up to $>80 \%$ [5-9]. Therefore, this polymer solar cell is still being intensively studied as a benchmark. Thereafter, owing to syntheses of various new materials, optimization of blend morphology, and development of new device structures, a certified PCE in excess of $8 \%$ has been listed in the solar cell efficiency table (version 37) [14]. In 2011, a PCE of 9.2\% has been reported [15]. Now it is just a matter of time before PCE of polymer solar cells will exceed $10 \%$.

For the improvement of the device efficiency, new materials and device structures are developed mainly on the basis of macroscopic properties of $J-V$ 
characteristics. However, the $J-V$ characteristics just provide us the final result of a series of fundamental photovoltaic conversion events including photon absorption, exciton generation, exciton migration, charge separation, charge recombination, charge dissociation, charge transport, and charge collection. In other words, the essential problem causing a poor device performance cannot be specified only from the $J-V$ characteristics. Thus, it is of particular importance to elucidate each fundamental event to design new materials and develop new device structures rationally. Photovoltaic conversion events occur in a range of over nine orders of magnitude on a temporal scale from $\sim 10^{-14} \mathrm{~s}$ for ultrafast charge separation to $\sim 10^{-5} \mathrm{~s}$ for charge collection to the electrode. Transient absorption spectroscopy is therefore a powerful tool for studying such conversion events in photovoltaic devices directly. By analyzing the transient spectra in detail, we can assign all the transient species such as exciton, polaron pair, free polaron, and trapped polaron separately and discuss their dynamics quantitatively [16-21]. This chapter describes the assignment of excitons and charge species and the photophysics of photovoltaic conversion events in polymer solar cells studied by transient absorption spectroscopy.

\section{Temporal Scale of Photovoltaic Conversion}

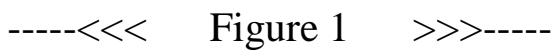

Here we describe the temporal scale of photovoltaic conversion events in polymer solar cells. Figure 1 shows a schematic illustration of the most simple polymer solar cells with a bilayered structure of the hole-transporting (donor) and 
electron-transporting (acceptor) materials, which is similar to silicon-based solar cells consisting of p-type and n-type semiconductors. In polymer solar cells, as shown in the figure, the photon absorption first produces singlet excitons that are electron-hole pairs tightly bound by the Coulomb attraction. This exciton generation results from an electronic transition from the ground state to an excited state due to the photon absorption, which typically occurs on a time scale of femtosecond $\left(\sim 10^{-15} \mathrm{~s}\right)$. In contrast, the photon absorption in silicon-based solar cells produces not excitons but freely mobile charge carriers directly at room temperature. This is the most critical difference between them, which results from the lower dielectric constant and larger effective mass (lower charge carrier mobility) in organic semiconductors than in inorganic semiconductors [22]. A critical distance $r_{\mathrm{C}}$ at which the thermal energy of a charge carrier is equal to the Coulomb attractive potential energy [23] would be as long as $14-19 \mathrm{~nm}$ in organic materials at room temperature because dielectric constant is small $(\varepsilon=3-4)$, while it would be only $5 \mathrm{~nm}$ in crystalline silicon with a high dielectric constant $(\varepsilon=11.9)$. Furthermore, excitons are typically localized in organic materials as Frenkel excitons or charge transfer (CT) excitons while they are delocalized in crystalline silicon as Wannier excitons with a radius much larger than the lattice spacing [23]. Consequently, excitons generated in organic materials cannot be dissociated into free carriers at room temperature. Instead, they can migrate randomly in films before deactivating to the ground state (exciton diffusion). As a result, some excitons can reach a donor/acceptor interface where they can be dissociated into free carriers. The diffusion time is limited by the lifetime of excitons (typically $<1 \mathrm{~ns}$ ) and is dependent on the phase-separated structures in donor/acceptor blend films. In a finely mixed donor/acceptor blend, no exciton diffusion is required to generate free carriers. In a 
largely phase-separated blend such as RR-P3HT:PCBM annealed films, the exciton diffusion is observed on a time scale of tens picoseconds $\left(\sim 10^{-11} \mathrm{~s}\right)$ as described in section 5.1. At the donor/acceptor interface, excitons can be separated into the electron on the acceptor material (radical anion) and the hole on the donor material (radical cation or polaron) if the energy gap at the interface is enough to break the Coulomb attraction. The charge separation is promptly completed in the order of $\sim 10^{-14} \mathrm{~s}[19,24]$. The electron and hole pair generated at the interface is often called bound radical pairs to be distinguished from tightly bound electron-hole pairs of excitons in the bulk. Some bound radical pairs can be dissociated into free carriers (charge dissociation) in competition with the geminate recombination to the ground state or triplet state (charge recombination) on a time scale of $10^{-12}$ to $10^{-9} \mathrm{~s}$ [19]. The dissociated free carriers are transported to each electrode through repetitive charge hoppings in an energetically disordered matrix (charge transport). Some of them escaping from the bimolecular recombination are collected to the electrode (charge collection). The charge collection time is dependent on the charge mobility, the thickness of the active layer, and the electric field applied to the layer. Recent studies have shown that it takes $10^{-6}$ to $10^{-5} \mathrm{~s}$ for charge carriers to be collected to the electrode [25]. Finally, the photocurrent is generated as a result of the series of photovoltaic conversion events. In other words, the device performance of $J-V$ characteristics is just the final result of the series of photovoltaic conversion events ranging from $10^{-14}$ to $10^{-5} \mathrm{~s}$ (nine orders of magnitude on a temporal scale). In this chapter, we focus on such rapid photovoltaic conversion events studied by transient absorption spectroscopy and discuss the findings obtained from the kinetics analysis. 


\section{Transient Absorption Spectroscopy}

Transient absorption spectroscopy is the most useful method for directly observing photovoltaic conversion events ranging from $10^{-14}$ to $10^{-5} \mathrm{~s}$. However, it is difficult to measure the absorption of thin films such as polymer solar cells where the active layer is typically as thin as $100 \mathrm{~nm}\left(=10^{-5} \mathrm{~cm}\right)$. For example, the absorbance would be as small as $10^{-5}$ in the case of a molar absorption coefficient of $10^{4} \mathrm{M}^{-1} \mathrm{~cm}^{-1}$, which is a typical value for organic dye molecules, a molar concentration of $0.1 \mathrm{mM}$, and an optical path length of $10^{-5} \mathrm{~cm}$. To detect an absorbance change of $10^{-5}$, it is necessary to measure only $1 / 50000$ of the change in the optical probe signal separately from various noises.

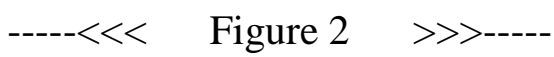

Figure 2 shows a block diagram of the highly sensitive microsecond transient absorption spectroscopy system [17]. In this system, a probe light is provided from a tungsten lamp with a power source stabilized to reduce fluctuation of the probe intensity. To reduce unnecessary scattering light, stray light, and emission from the sample, two monochromators and appropriate optical cut-off filters are placed before and after the sample. An excitation light is supplied from a dye laser pumped by a nitrogen laser, which can excite the absorption peak of thin-film samples to give a high yield of photoexcitations. The probe light passing through the sample is detected with a PIN photodiode such as Si or InGaAs depending on the measuring wavelength. The signal from the photodiode is pre-amplified and sent to the main amplification system with 
electronic band-pass filters to improve the signal to noise ratio. The amplified signal is collected with a digital oscilloscope, which is synchronized with a trigger signal of the laser pulse from a photodiode. In our system, the detectable absorbance change is as small as $10^{-5}$ to $10^{-6}$ depending on the measuring time domain after appropriate accumulation owing to the amplification and noise reduction system.

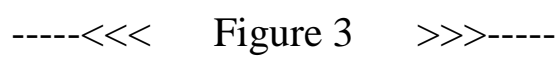

For ultrafast phenomena on a time scale of $<1 \mathrm{~ns}$, the pump and probe method is widely employed. In this method, ultrashort laser pulses serve as the pump light for the sample excitation and the probe light for the transmittance measurement. Transient absorption can be measured at various delay times by controlling the arrival time of each laser pulse at the sample. The arrival time of each laser pulse can be tuned with an optical delay line: the probe light is delayed relative to the pump light because of the additional path length in the optical delay line. For example, when the total optical delay length is set at $3 \mathrm{~cm}$ the probe light is delayed by 100 ps relative to the pump light, and therefore the transmittance at 100 ps after the laser excitation can be measured. Figure 3 shows a block diagram of a typical pump and probe spectroscopy system we employ [17-21]. This system consists of a transient absorption spectrometer and a regenerative amplified Ti:sapphire laser. The amplified Ti:sapphire laser provided $800-\mathrm{nm}$ fundamental pulses at a repetition rate of $1 \mathrm{kHz}$ with an energy of $0.8 \mathrm{~mJ}$ and a pulse width of 100 fs (FWHM), which were split into two optical beams with a beam splitter to generate pump and probe pulses. One fundamental beam was converted into pump pulses at $400 \mathrm{~nm}$ with a second harmonic generator or pump pulses at other 
wavelengths with an ultrafast optical parametric amplifier. The other fundamental beam was converted into white light continuum pulses employed as probe pulses over the wide wavelength from 400 to $1700 \mathrm{~nm}$. The pump pulses were modulated mechanically with a repetition rate of $100 \mathrm{~Hz}$ for visible and $500 \mathrm{~Hz}$ for near-IR measurements. The temporal evolution of the probe intensity was recorded with a Si CCD-array photodetector for the visible measurement and with an InGaAs digital line scan camera for the near-IR measurement. Transient absorption spectra and decays were collected over the time range from -5 ps to 3 ns. Typically, 200-1000 laser shots were averaged on each delay time to obtain a detectable absorbance change as small as $10^{-4}-10^{-3}$ depending on the monitor wavelength. The polarization direction of the linearly polarized probe pulse was set at a magic angle of $54.7^{\circ}$ with respect to that of the pump pulse to cancel out orientation effects on the dynamics. In order to measure further the transient absorption in a longer time range, a longer optical delay line would be required. Alternately, additional laser is employed to provide delayed probe pulses with an electric delay generator synchronized with the pump laser pulse [26]. Note that it is necessary to correct signal jitters due to the electric circuit.

\section{Assignment of photoexcitations}

Let us first describe how to assign transient species such as singlet and triplet excitons and polarons generated in polymer solar cells. Here, we explain the assignments on the basis of our recent studies [16-20].

\subsection{Singlet and Triplet Excitons}


Singlet and triplet excitons can be often observed for pristine films upon photoexcitation. In particular, singlet excitons can be easily observed as the initial product by photon excitation because most conjugated polymers have $S_{0} \rightarrow S_{n}$ spin-allowed absorption bands from the singlet ground state. In contrast, it is difficult to detect triplet excitons for highly emissive conjugated polymer films such as polyfluorene because the intersystem crossing yield is low due to small spin-orbit coupling. On the other hand, triplet excitons can be observed for conjugated polymer films with high intersystem crossing yield such as regiorandom poly(3-hexylthiophene) (RRa-P3HT).

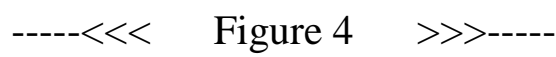

Figure 4a shows transient absorption spectra of RRa-P3HT pristine films. Immediately after the laser excitation, a large absorption band is observed at around $1000 \mathrm{~nm}$. This band is ascribable to singlet exciton because the decay constant is consistent with the lifetime of fluorescence measured by the time-correlated single photon counting (TC-SPC) method. Note that the averaged decay constant measured by transient absorption is typically shorter than the lifetime measured by the TC-SPC method because singlet-singlet exciton annihilation is involved in the transient absorption measurement with excitation intensity much higher than that in the TC-SPC measurement. Thus, a longer decay fraction independent of the excitation intensity should be extracted by changing the excitation intensity. If it is in agreement with the fluorescence lifetime, the initial product can safely be assigned to singlet exciton.

As shown in Figure 4a, the singlet exciton band at around $1000 \mathrm{~nm}$ disappears 
at 100 ps after the laser excitation, and instead a small absorption band is observed at around $800 \mathrm{~nm}$. This is indicative of the interconversion from singlet to triplet excitons. Figure $4 \mathrm{~b}$ shows transient absorption spectra of RRa-P3HT pristine films on a time scale of microseconds, which is similar to the long-lived species observed on a time scale of nanoseconds mentioned above. As shown in the inset to the figure, the transient signal decays monoexponentially with a lifetime of $7 \mu$ s under a nitrogen atmosphere while it decays more rapidly under an oxygen atmosphere. Molecular oxygen is a general and efficient quencher of singlet and triplet excitons in organic materials [27]. Thus, such a long lifetime is ascribable to triplet excitons because it is too long to be assigned to singlet excitons. Although electron spin resonance (ESR) is a useful method for distinguishing between singlet and triplet states as reported in the literatures [28-30], the oxygen quenching is most widely employed to assign triplet excitons. Note that not all triplet excitons are quenched by molecular oxygen because quenching is a diffusion-limited reaction. Roughly speaking, triplet excitons with a long lifetime ( $>\mu \mathrm{s})$ can be effectively quenched by molecular oxygen even in solid films depending on the oxygen permeability of the film.

\section{$-----<<<\quad$ Figure $5 \quad>>>-----$}

In most cases, as described above, singlet excitons are observed as the initial product by photon absorption. However, this is not true for regioregular P3HT (RR-P3HT) pristine films excited by intense laser pulses. Figure 5 shows the transient absorption spectra of RR-P3HT pristine films under different excitation conditions. At an excitation intensity of $15 \mu \mathrm{J} \mathrm{cm}^{-2}$, a large absorption band is observed at around 1200 
$\mathrm{nm}$ immediately after the laser excitation. As the excitation intensity is increased from 15 to $120 \mu \mathrm{J} \mathrm{cm}^{-2}$, this band disappears and instead a new absorption band is observed at around $1000 \mathrm{~nm}$. From the intensity dependence of the initial transient signals, we ascribe the 1200-nm band to a singlet exciton and the 1000-nm band to polarons. At higher excitation intensities, polarons are generated even at 0 ps from hot exciton states formed by the singlet-singlet exciton annihilation. Note that no exciton diffusion is involved in the singlet-singlet exciton annihilation because the duration is too short. The energy of two photons at $400 \mathrm{~nm}$ corresponds to $6.2 \mathrm{eV}$, which is much larger than the ionization potential of RR-P3HT films. Thus, it would be more appropriate to assign the polaron formation to two-photon ionization. This finding suggests that the initial product by photon absorption is not always a singlet exciton particularly at a high excitation intensity. In conclusion, for the assignment of singlet excitons, it is essential to analyze not only the decay kinetics but also the intensity dependence of the spectrum and kinetics.

\subsection{Polarons}

As mentioned above, polarons are efficiently generated from excitons at a donor/acceptor interface. Thus, they can be more clearly observed in polymer:fullerene blend films rather than in pristine conjugated polymer films. Some polarons escaping from the geminate recombination at the interface can survive up to a time scale of microseconds or more. On such a longer time scale, therefore, polarons can often be observed separately from singlet and triplet excitons. Figure 6a shows the transient absorption spectra of RRa-P3HT:PCBM blend films from 0 to 3 ns after the laser excitation. In this time domain, the absorption spectrum varies with time, 
suggesting that the transient species changes with time. As will be described in detail later, there are spectral overlaps among P3HT singlet excitons, P3HT polarons, and PCBM radical anions at an early time stage. On the other hand, as shown in Figure 6b, no change in transient absorption spectra is observed for RRa-P3HT:PCBM blend films on a time scale of microseconds, suggesting that transient species remain the same. The large absorption band at $900 \mathrm{~nm}$ and the small and sharp absorption band at 1030 nm are safely ascribed to P3HT hole polaron and to PCBM radical anion, respectively. Both bands exhibit power-law decay dynamics with an exponent of 0.35 over a long time range up to milliseconds. The power-law decay is characteristic of the bimolecular recombination of long-lived, dissociated charge carriers. The subunity value for the exponent suggests trap-limited bimolecular recombination of polarons in an energetically disordered matrix while the exponent is unity for trap-free bimolecular recombination [31].

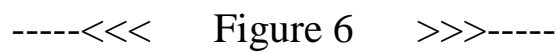

Figure 7a shows the transient absorption spectra of RR-P3HT:PCBM blend films from 0 to 3 ns after the laser excitation. In this time domain, as in the case with RRa-P3HT:PCBM blend films, the absorption spectrum varies with time. The large absorption band at around $1250 \mathrm{~nm}$ is in good agreement with that observed immediately after the laser excitation of RR-P3HT pristine films as described above, and therefore can be ascribed to P3HT singlet exciton. As shown in Figure 7b, the broad absorption bands at 700 and $1000 \mathrm{~nm}$ are still observed on a time scale of microseconds. Interestingly, these two bands exhibit the power-law decay dynamics 
with different exponents, which remain the same under an oxygen atmosphere. Thus, they can be ascribed to P3HT polarons but must be different polarons as will be described in detail in section 6.3. In other words, this spectral change shows the formation of P3HT polaron from P3HT singlet exciton.

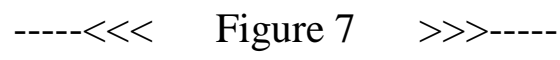

In the two cases described above, polarons are efficiently generated from singlet excitons and therefore can be separately observed on a time scale of microseconds. In some cases, however, a triplet exciton band overlaps with a polaron band. Here we show an example of simultaneous observation of triplet excitons and polarons. Figure 8a shows transient absorption spectra of a blend film of poly[(4,4'-didecyl[2,2'-bithiophene]-5,5'-diyl)-1,4-phenylene $\left(\mathrm{PT}_{10} \mathrm{PhT}_{10}\right)$ and PCBM. A large absorption band is observed at $700 \mathrm{~nm}$ with a shoulder at around $850 \mathrm{~nm}$ at 10 $\mu$ s after the laser excitation. The absorption spectrum varies with time, with the absorption peak shifting from 700 at $10 \mu$ s to $900 \mathrm{~nm}$ for time delays longer than 100 $\mu$ s. This spectral change suggests that there are two different transient species in the blend film. Figure $8 \mathrm{~b}$ shows transient absorption decays of $\mathrm{PT}_{10} \mathrm{PhT}_{10}: \mathrm{PCBM}$ blend films monitored at $700 \mathrm{~nm}$. This decay dynamics can be fitted with the sum of a single exponential function and a power-law equation. As shown in the figure, the exponential decay component is quenched under oxygen atmosphere, and therefore ascribed to triplet exciton. On the other hand, the exponent of the power-law decay remains the same even under oxygen atmosphere, and therefore is ascribed to polymer polarons. In conclusion, for the assignment of polarons, it is essential to observe 
polarons separately from singlet and triplet excitons. Singlet excitons can be excluded by transient absorption measurements on a time scale of microseconds. In order to distinguish between triplet excitons and polarons, it is useful to analyze the decay kinetics and the oxygen quenching measurement. Triplet excitons generally decay monoexponentially while polarons typically exhibit the power-law decay dynamics on a time scale of microseconds. In the presence of molecular oxygen, the lifetime of triplet excitons is effectively shortened but the decay dynamics of polarons does not change.

$-----<<<\quad$ Figure $8 \quad>>>-----$

\subsection{Other Charge Carriers}

In order to assign charge carriers of unknown or new materials, it is necessary to measure the absorption spectrum and to quantitatively evaluate the molar absorption coefficient of each carrier separately using a model system with known donor or acceptor materials. Here we show an example of the assignment of PCBM anion by using tetramethyl-p-phenylenediamine (TMPD) as a known electron donor. Figure 9 shows transient absorption spectra of a polystyrene film doped with TMPD and PCBM. Two absorption bands are observed at 570 and $1020 \mathrm{~nm}$ after the laser excitation. The absorption band at $570 \mathrm{~nm}$ is in good agreement with that reported for the oxidation product of TMPD called Wurster's Blue [32], and is therefore safely assigned to the TMPD radical cation. As shown in the inset to the figure, both bands exhibit the power-law decay dynamics with the same exponent on a longer time scale (>10 ps), 
indicating the bimolecular recombination without other decay pathways. In other words, no other transient species such as singlet and triplet excitons contribute to the transient absorption spectra. Thus, the absorption band at $1020 \mathrm{~nm}$ is assigned to the PCBM radical anion, which is consistent with that of radical anions of other fullerene derivatives: a $C_{60}$ radical anion $(1080 \mathrm{~nm})$, a methanofullerene radical anion $(1040 \mathrm{~nm})$, and a fulleropyrrolidine radical anion $(1010 \mathrm{~nm})$ [33]. Furthermore, the molar absorption coefficient of the PCBM radical anion can be evaluated to be $\varepsilon=6000 \mathrm{M}^{-1}$ $\mathrm{cm}^{-1}$ at $1020 \mathrm{~nm}$ on the basis of that of the TMPD radical cation $\left(\varepsilon=12000 \mathrm{M}^{-1} \mathrm{~cm}^{-1}\right)$ [32,34]. In conclusion, it is useful to employ known donor or acceptor materials for the assignment of unknown charge carriers. Note that it important to analyze the spectrum and the dynamics carefully to confirm that there is no contribution of other species.

$-----<<<\quad$ Figure $9 \quad>>>-----$

\section{Exciton Dynamics}

Here, we focus on the dynamics of singlet and triplet excitons mainly in pristine polymer films [18] and also describe the energy transfer in a polymer/polymer blend [21].

\subsection{Exciton Delocalization}

As mentioned above, the absorption band of singlet excitons in P3HT films is red-shifted with time, suggesting delocalization of singlet excitons. Figure 10 summarizes the transient absorption spectra of RRa-P3HT and RR-P3HT pristine films. 
The singlet exciton band is red-shifted from 900 to $1060 \mathrm{~nm}$ for RRa-P3HT and from 1200 to $1250 \mathrm{~nm}$ for RR-P3HT from 0 through $100 \mathrm{ps}$ after the excitation at $400 \mathrm{~nm}$. Such a peak shift suggests that singlet excitons efficiently migrate on a time scale of picoseconds, resulting in the exciton delocalization into longer conjugated segments and the singlet-singlet exciton annihilation under an intense excitation. The stabilization energy corresponds to $\Delta E=0.21 \mathrm{eV}$ for RRa-P3HT and $\Delta E=0.04 \mathrm{eV}$ for RR-P3HT. The large $\Delta E$ for RRa-P3HT is probably because RRa-P3HT amorphous films have a relatively wide distribution of energetic disorders compared to RR-P3HT crystalline films. Interestingly, as shown in Figure 10c, no peak shift is observed for RR-P3HT films excited at $620 \mathrm{~nm}$ that is the absorption edge of RR-P3HT. The absorption band is already observed at $1250 \mathrm{~nm}$ immediately after the laser excitation and still observed at $1250 \mathrm{~nm}$ at $100 \mathrm{ps}$. This finding shows that delocalized P3HT singlet excitons in crystalline domains can be selectively observed by selective excitation at $620 \mathrm{~nm}$. As reported in the literatures [35-37], the exciton diffusion constant can be evaluated by analyzing the dynamics of singlet-singlet exciton annihilation under different excitation intensities.

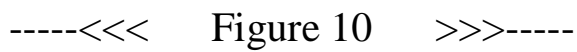

As the excitation intensity increases further, the singlet-singlet exciton annihilation is observed even at 0 ps because singlet excitons are likely to be generated in proximity to each other at high concentrations without diffusion. The threshold intensity is $7 \times 10^{18} \mathrm{~cm}^{-3}$ for RRa-P3HT films excited at $400 \mathrm{~nm}, 3 \times 10^{18} \mathrm{~cm}^{-3}$ for RR-P3HT films excited at $400 \mathrm{~nm}$, and $8 \times 10^{17} \mathrm{~cm}^{-3}$ for RR-P3HT films excited at 620 
nm. Assuming a three-dimensional sphere without taking their anisotropic distribution into consideration, an averaged interaction radius of two excitons at 0 ps can be estimated to be $\sim 3.2 \mathrm{~nm}$ for RRa-P3HT films excited at $400 \mathrm{~nm}, \sim 4.3 \mathrm{~nm}$ for RR-P3HT films excited at $400 \mathrm{~nm}$, and $\sim 6.7 \mathrm{~nm}$ for RR-P3HT films excited at $620 \mathrm{~nm}$. The difference in the interaction radius is indicative of the difference in the exciton delocalization at 0 ps. In other words, singlet excitons are more delocalized in RR-P3HTcrystalline films than in RRa-P3HT amorphous films and upon the excitation close to the bandgap than upon the excitation above the bandgap. These are consistent with the peak wavelengths of the singlet exciton band as discussed above.

\subsection{Energy Transfer}

In some donor/acceptor blends, the efficient energy transfer from photogenerated singlet excitons is observed before the charge generation at the interface. Here we describe the energy transfer in polymer/polymer blends of RR-P3HT and poly(9,9-dioctylfluorene-alt-benzothiadiazole) (F8BT), which have a large spectral overlap between the fluorescence of F8BT and the absorption of RR-P3HT. Figure 11a shows the transient absorption spectra of RR-P3HT:F8BT blend films excited at $400 \mathrm{~nm}$ where $60 \%$ of photons are absorbed by F8BT and the remaining $40 \%$ are absorbed by RR-P3HT. The transient absorption spectra in a picosecond can be well reproduced by the sum of the S-S absorption spectrum observed for each pristine film of F8BT and RR-P3HT, suggesting that the major transient species are F8BT and RR-P3HT singlet excitons in the time domain of $<1$ ps. In other words, the broad absorption at around $1000 \mathrm{~nm}$ observed at $0 \mathrm{ps}$ is ascribed to F8BT singlet excitons. The large absorption at around $1250 \mathrm{~nm}$ observed after $1 \mathrm{ps}$ is ascribed to RR-P3HT 
singlet excitons. On the basis of the spectral simulation, the decay dynamics of each transient species can be obtained as shown in Figure 11b. The F8BT singlet exciton decays monoexponentially with a lifetime of $\sim 0.3$ ps. On the other hand, $\sim 40 \%$ of RR-P3HT singlet excitons are promptly generated immediately after the laser excitation and the other $\sim 60 \%$ of RR-P3HT singlet excitons are generated with a rise constant of $\sim 0.3$ ps. The prompt generation of RR-P3HT singlet excitons is ascribed to the direct excitation of RR-P3HT at $400 \mathrm{~nm}$. The delayed generation of RR-P3HT singlet excitons is ascribed to the energy transfer from F8BT singlet excitons because the rise time of RR-P3HT singlet excitons is in agreement with the decay constant of F8BT singlet excitons. Such rapid energy transfer within a picosecond suggests that all F8BT singlet excitons are efficiently transferred into RR-P3HT domains without exciton diffusion in the blend film. In other words, there are no large pure domains in the blend: F8BT is molecularly dispersed in RR-P3HT domains and/or RR-P3HT is dispersed in F8BT rich domains. This is probably because chloroform is so rapidly evaporated that largely phase-separated pure domains cannot be formed. On the basis of the Förster theory assuming point dipoles, such rapid energy transfer is possible at a separation distance of $\sim 0.9 \mathrm{~nm}$ between F8BT and RR-P3HT. Consequently, at least $60 \%$ of RR-P3HT singlet excitons are located near the interface of RR-P3HT/F8BT because of the efficient energy transfer from F8BT singlet excitons. This would be beneficial for the subsequent charge separation but the charge generation efficiency is actually not as high as that in RR-P3HT:PCBM blends. This may be due to the difference in the interfacial structures [21].

$----<<<\quad$ Figure $11 \quad>>>-----$ 


\subsection{Triplet Formation}

Triplet excitons are generally converted through the intersystem crossing from the lowest singlet exciton. As mentioned in section 4.1 (see Figure 4), triplet excitons are generated from singlet excitons in RRa-P3HT films. Indeed, as shown in Figure 12 , the triplet rise $(\sim 4 \mathrm{ps})$ is in good agreement with the singlet decay ( $\sim 4 \mathrm{ps})$, suggesting that triplet excitons are rapidly converted from singlet excitons. The rise and decay time is dependent on the excitation intensity, suggesting that the singlet exciton-exciton annihilation contributes to the rapid interconversion. Interestingly, the formation yield of triplet excitons is estimated to be as high as $~ 25 \%$ at 10 ps from the decay analysis $[18,38]$, even though singlet excitons are significantly quenched by the singlet exciton-exciton annihilation. If triplet excitons were formed via the intersystem crossing from relaxed singlet excitons, the triplet yield at 10 ps should be as small as $\sim 1 \%$ because the intersystem crossing rate has been reported to be $\sim 1 \mathrm{~ns}^{-1}$ for poly(3-octylthiophene) in a xylene solution [38]. Therefore, such a short interconversion time of $\sim 4$ ps cannot be simply explained in terms of the intersystem crossing from the lowest singlet exciton state to triplet exciton state. Rather the rapid triplet formation results from a higher singlet exciton state generated by the singlet exciton-exciton annihilation in the picosecond time domain. The triplet formation from a higher exciton state should be completed before the relaxation to the lowest singlet exciton state, because the interconversion in the relaxed exciton states is limited by the slow intersystem crossing rate. We therefore conclude that the rapid triplet formation is in competition with the vibrational relaxation to the lowest exciton or 
polaron pair states within $<100$ fs. Such a rapid triplet formation is indicative of efficient spin-mixing between singlet and triplet excitons. Higher exciton states in conjugated polymers are likely to be more mixed with CT states, resulting in a relatively longer electron-hole separation and hence a smaller electron exchange integral $2 J$. The small energy gap of $2 J$ between the singlet and triplet states would promote the interconversion between them. For a very small exchange integral, hyperfine interaction (HFI) between the electron and nuclear spins generally plays an important role in the interconversion mechanism. In organic radicals, the HFI energy is typically in the order of $\sim 5 \mathrm{mT}$, which corresponds to an interconversion time of several nanoseconds $[39,40]$. This is consistent with an interconversion time of $\sim 1$ ns reported for poly(3-octylthiophene) in a xylene solution [38]. Thus, the ultrafast triplet formation on a short time scale of picoseconds suggests that the interconversion mechanism is different from the normal intersystem crossing from the lowest singlet exciton.

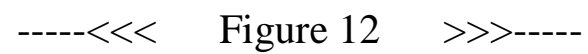

Fission of singlet excitons into two triplet exciton pairs is spin-conserving and hence spin-allowed [23]. Singlet fission is primarily induced by spin dipole-dipole interaction while the normal intersystem crossing is induced by the spin-orbit coupling [41]. Triplet formation from singlet fission has been reported for molecular crystals [42-47], and also for conjugated polymer films [48-50]. The energy of the two triplet pair state ${ }^{1}(\mathrm{TT})$ is approximated by twice the energy of isolated triplet excitons $\left(2 E_{\mathrm{T} 1}\right)$. This is in good approximation for molecular crystals with weak intermolecular 
interactions. Thus, $2 E_{\mathrm{T} 1}$ is generally considered to be the threshold energy for singlet fission. Assuming that the energy difference between the lowest singlet and triplet states $\Delta E_{\mathrm{ST}}$ is $0.7 \mathrm{eV}$ for RRa-P3HT, which is a typical value for various amorphous conjugated polymers [51,52], the energy level of the lowest triplet exciton state $\left(E_{\mathrm{T} 1}\right)$ is roughly estimated to be $1.6 \mathrm{eV}$ for RRa-P3HT amorphous films. Therefore, the threshold energy for singlet fission is estimated to be $3.2 \mathrm{eV}$ for RRa-P3HT, which is slightly higher than the excitation energy $(3.1 \mathrm{eV})$. In other words, the singlet fission by one photon excitation at $400 \mathrm{~nm}$ is thermodynamically unfavorable for RRa-P3HT. Indeed, no distinct triplet signal is observed for RRa-P3HT films immediately after the laser excitation under lower excitation intensities. On the other hand, singlet fission is thermodynamically possible from a higher singlet exciton state generated by the singlet exciton-exciton annihilation (singlet fusion). If triplet excitons are generated from singlet fission, the back recombination of the two triplet pair state ${ }^{1}(\mathrm{TT})$ to the singlet exciton state is also spin-allowed and therefore expected to be much faster than the normal spin-forbidden transition from isolated triplet excitons to the ground state. As shown in Figure 12, the lifetime of triplet signals observed for RRa-P3HT is as short as 300 ps, which is much faster than that of "isolated" triplet excitons $(\sim 7 \mu$ s). This rapid decay is ascribed to the recombination of triplet exciton pairs to the singlet exciton state. We therefore conclude that triplet excitons observed for RRa-P3HT films are mainly generated through the singlet fission from a higher singlet exciton state produced by the singlet exciton-exciton annihilation (singlet fusion followed by singlet fission into triplet exciton pairs). Note that no triplet formation is observed for RR-P3HT crystalline films even though singlet fission is thermodynamically possible from a higher singlet exciton state. This is probably because the formation of polarons or 
polaron pairs is more efficient than that of the singlet fission because of the larger interchain interaction in highly ordered crystalline RR-P3HT films.

Finally, we note the relevance of singlet fission to polymer solar cells. As mentioned above, singlet fission produces two triplet excitons from one singlet exciton. This is analogous to multiple exciton generation in semiconductor quantum dots $[53,54]$, which has attracted increasing interest because more than one excitons could be generated by one photon absorption. Recent studies have demonstrated that the singlet fission indeed contributes to the photocurrent in pentacene/ $\mathrm{C}_{60}$ bilayered films $[55,56]$. The singlet fission in RRa-P3HT films is not directly linked with the polaron formation. This is partly because the triplet exciton state is located lower in energy than the polaron state. Although further studies are needed for efficient singlet fission into triplet excitons followed by efficient charge generation, it is a challenging and attractive target to develop polymer solar cells based on singlet fission.

\section{Charge Dynamics}

Let us move on to the dynamics of charge species generated in polymer:fullerene blends. In particular, we focus on the charge dynamics in P3HT:PCBM blends and summarize the relevance to the device performance $[19,20]$.

\subsection{Charge Generation}

In RRa-P3HT:PCBM blend films, as shown in Figure 6a, P3HT singlet exciton is already quenched to $\sim 50 \%$ even at 0 ps, and disappears rapidly in a picosecond, followed by P3HT polaron generation. Figure 13a shows the time evolution of P3HT singlet excitons and polarons in the RRa-P3HT:PCBM blend film. The P3HT singlet 
exciton decays monoexponentially with a time constant of 0.2 ps. On the other hand, more than half of the polarons are promptly generated even at $0 \mathrm{ps}$ and the remaining polarons are also rapidly generated with the same rise constant of $0.2 \mathrm{ps.} \mathrm{This}$ agreement suggests that polarons are efficiently generated from P3HT singlet excitons in a picosecond. The rapid formation of polarons is ascribed to the prompt charge generation at the interface of RRa-P3HT/PCBM, because the exciton migration is negligible on such a short time scale $(<0.2 \mathrm{ps})$. This is probably because PCBM molecules are more homogeneously dispersed in RRa-P3HT amorphous films than in RR-P3HT crystalline films. Assuming a homogeneous distribution of $50 \mathrm{wt} \%$ PCBM in RRa-P3HT films, the intermolecular distance of neighboring PCBM molecules would be less than $1 \mathrm{~nm}$. As mentioned in section 5.1, the delocalization radius of singlet excitons is estimated to be $3.2 \mathrm{~nm}$ in the RRa-P3HT film. This suggests that singlet excitons generated can promptly encounter a PCBM molecule without exciton migration. Furthermore, as shown in Figure 13b, no decay is observed for the photobleaching at $470 \mathrm{~nm}$, suggesting that all the singlet excitons are converted to polarons without deactivating to the ground state. We therefore conclude that both the exciton diffusion efficiency $\left(\eta_{\mathrm{ED}}\right)$ and the charge transfer efficiency $\left(\eta_{\mathrm{CT}}\right)$ are as high as 100\% in RRa-P3HT:PCBM blend films.

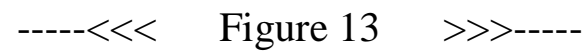

In RR-P3HT:PCBM blend films, as shown in Figure 7a, P3HT singlet excitons are also already quenched to $\sim 50 \%$ even at 0 ps, and decays on a time scale of picoseconds, followed by efficient P3HT polaron generation. Figure 14 shows the 
time evolution of the singlet exciton band at $1200 \mathrm{~nm}$ and the polaron band at $720 \mathrm{~nm}$. The decay of P3HT singlet excitons can be fitted by a double exponential function with a short lifetime of $\sim 10$ ps and an intrinsic exciton lifetime of 330 ps. On the other hand, a part of P3HT polarons are promptly generated even at 0 ps and the others are gradually generated with the same time constant as the short lifetime of P3HT singlet excitons. The delayed polaron formation ( $10 \mathrm{ps})$ is much slower than that observed for RRa-P3HT:PCBM blend films ( $<1 \mathrm{ps})$. Furthermore, the rise and decay constants depend on the P3HT domain size: both increase with increasing P3HT concentration and slightly increase after the thermal annealing. We therefore assign the delayed formation to the polaron generation via the exciton migration to the interface of RR-P3HT/PCBM. In other words, the time constant of the delayed formation of polarons is limited by the exciton migration in relatively large crystalline domains of RR-P3HT. This is consistent with the recent transient studies $[57,58]$. On the other hand, the prompt polaron formation $\left(>10^{13} \mathrm{~s}^{-1}\right)$ is ascribed to the charge generation at the interface of RR-P3HT/PCBM. The rate constant of the prompt polaron formation is $10^{4}$ times faster than the deactivation rate constant $\left(3.0 \times 10^{9} \mathrm{~s}^{-1}\right)$ of singlet excitons in RR-P3HT pristine films. Thus, the charge transfer efficiency $\left(\eta_{\mathrm{CT}}\right)$ is estimated to be $\sim 100 \%$ at the interface of RR-P3HT/PCBM. Because $\eta_{\mathrm{CT}} \approx 100 \%$, the exciton diffusion efficiency is estimated to be $\eta_{\mathrm{ED}}=\eta_{\mathrm{q}} \eta_{\mathrm{CT}} \approx \eta_{\mathrm{q}}=k_{\mathrm{q}} /\left(k_{\mathrm{F}}+k_{\mathrm{q}}\right)=93 \%$ before the thermal annealing and 89\% after the thermal annealing in RR-P3HT:PCBM blend films. In other words, there is almost $10 \%$ loss in $\eta_{\mathrm{ED}}$ in either case although it is still high enough to collect singlet excitons into the interface of RR-P3HT/PCBM.

$-----<<<\quad$ Figure $14 \quad>>>-----$ 


\subsection{Geminate and Bimolecular Recombination}

The charge recombination between electrons and holes can be typically classified into monomolecular (geminate) recombination and bimolecular recombination. It is important to distinguish between the two because the photocurrent generation is critically dependent on whether the electron and hole are bound as a geminate pair at the interface or dissociated into free carriers. However, it is impossible to distinguish between geminate charge pairs and free carriers from transient absorption spectra alone, because the charge species are identical in either case. Geminate recombination is the first-order reaction, and hence the decay constant is, as shown in Figure 15a, independent of the concentration of the transient species. On the other hand, bimolecular recombination is the second-order reaction, and hence the half-life is, as shown in Figure 15b, dependent on the concentration of the transient species: it should be theoretically half at twice concentration. Therefore, we can distinguish whether electron and hole are bound as a geminate pair at the interface or dissociated into free carriers by analyzing the intensity dependence of the decay dynamics.

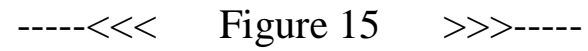

In RRa-P3HT:PCBM blend films, as described in section 6.1, polarons are promptly generated in a picosecond with $\eta_{\mathrm{ED}}=100 \%$. However, as shown in Figure 16, the PCBM anion band at $1030 \mathrm{~nm}$ decays monoexponentially to $30 \%$ with a time 
constant of $\sim 0.8 \mathrm{~ns}$. The photobleaching at $480 \mathrm{~nm}$ also recovers with the same constant of $\sim 0.8$ ns. Furthermore, as shown in Figure 17, all the decay dynamics of the P3HT polarons at $850 \mathrm{~nm}$, the PCBM anions at $1030 \mathrm{~nm}$, and the photobleaching at 480 $\mathrm{nm}$ are independent of the excitation intensity, and therefore can be ascribed to the monomolecular (geminate) recombination of P3HT polarons and PCBM anions. We therefore conclude that 70\% of P3HT polarons geminately recombine with PCBM anions to the ground state and the remaining $30 \%$ of polarons can be dissociated into free carriers. The dissociated polarons survive until longer time domains and hence can be observed on a time scale of microseconds as shown in Figure 6b. In other words, the charge dissociation efficiency is as low as $\eta_{\mathrm{CD}}=30 \%$ in RRa-P3HT:PCBM blend films.

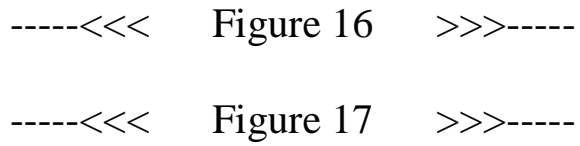

In RR-P3HT:PCBM blend films, there are two pathways for polaron generation: one is prompt generation $(<100 \mathrm{fs})$ at the interface and the other is delayed generation ( 10 ps) after the exciton migration to the interface. Here we focus on the nanosecond dynamics of two polarons bands: the delocalized polarons at $700 \mathrm{~nm}$ and localized polarons at $1000 \mathrm{~nm}$. This assignment is consistent with previous studies on P3HT pristine films where the 700-nm and 1000-nm bands are ascribed to interchain delocalized polarons and intrachain localized polarons, respectively $[13,29,30]$. As shown in Figure 18, the decay dynamics of the two bands is dependent on the excitation intensity at higher excitation intensities, indicating the bimolecular recombination of 
free polarons. We therefore conclude that all of the polarons at 700 and $1000 \mathrm{~nm}$ are ascribed to dissociated free polarons on a time scale of nanoseconds. In contrast to RRa-P3HT:PCBM blends, no decay is observed for RR-P3HT:PCBM blends even at lower excitation intensities. In other words, the charge dissociation efficiency is estimated to be as high as $\sim 100 \%$ for these two polarons. In addition, without going into detail, the other polaron in amorphous domains exhibits a charge dissociation efficiency of $38 \%$ before the thermal annealing, which is similar to that observed for RRa-P3HT:PCBM blends, and 69\% after the thermal annealing. Consequently, the overall charge dissociation efficiency is as high as $\eta_{\mathrm{CD}}=80 \%$ before the thermal annealing, and increases to $\eta_{\mathrm{CD}}=93 \%$ after the thermal annealing, which is three times larger than that for RRa-P3HT:PCBM blend films.

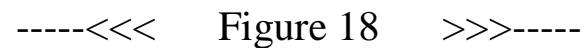

Such a high dissociation efficiency is consistent with the efficient device performance of RR-P3HT:PCBM solar cells. However, it cannot be rationally explained by the classical models such as those of Onsager [59] and Braun [60]. Recent theoretical studies demonstrate that the presence of donor/acceptor phase-separated interface increases the charge dissociation probability in comparison with the homogeneous blend [61-63]. Recently, Durrant pointed out the importance of considering the change in entropy associated with changing from a single exciton to two separated charges, by which the effective Coulomb capture radius is estimated to be $\sim 4$ $\mathrm{nm}$ at a typical donor/acceptor heterojunction [64]. This is much shorter than the Onsager radius $\left(r_{\mathrm{C}}=14-19 \mathrm{~nm}\right)$ in organic materials at room temperature as mentioned 
above. Interestingly, this effective Coulomb capture radius is comparable to our estimations of the delocalization radius of singlet excitons: singlet excitons with a radius of $~ 4.3-6.7 \mathrm{~nm}$ in RR-P3HT pristine films can be effectively dissociated into free polarons, while singlet excitons with a radius of $\sim 3.2 \mathrm{~nm}$ in RRa-P3HT pristine films form bound radical pairs. This correlation suggests that the separation distance of two charges at the interface is closely related to the delocalization radius of singlet excitons. On the other hand, Deibel and his coworkers have demonstrated that the efficient charge dissociation can be explained by kinetic Monte Carlo simulations considering delocalization of charge carriers within conjugated segments in polymer chain [65]. This is also consistent with our findings of the different delocalization radius of singlet excitons, because delocalized singlet excitons would convert to delocalized polarons. We therefore conclude that the longer separation distance of bound radical pairs $>4 \mathrm{~nm}$ can promote the dissociation of bound radical pairs and the formation of free polarons effectively, whereas the shorter separation distance of bound radical pairs $<4 \mathrm{~nm}$ cause in a significant loss due to the geminate recombination of bound radical pairs at the interface. In addition, desirable phase-separated structures in RR-P3HT:PCBM blend films can also promote the dissociation of bound radical pairs effectively whereas homogeneously-mixed blend structures in RRa-P3HT:PCBM blend films cause a significant loss due to the geminate recombination. Furthermore, the high charge mobility in RR-P3HT:PCBM blend films can also result in the efficient dissociation of bound radical pairs [60-66].

\subsection{Trap-Free and Trap-Limited Recombination}


As shown in Figure 7b, two absorption bands are clearly observed at around 700 and $1000 \mathrm{~nm}$ and decay slowly on a microsecond time scale. These two bands can be ascribed to polarons: the 700-nm band to delocalized polarons and the $1000-\mathrm{nm}$ band to localized polarons. Interestingly, the delocalized polaron band at $700 \mathrm{~nm}$ decays faster than the localized polaron band at $1000 \mathrm{~nm}$. The delocalized polaron band almost disappears and instead the localized polaron band is dominant at $100 \mu \mathrm{s}$. This is indicative of the different recombination dynamics between delocalized polaron and localized polaron. Figure 19 shows the transient absorption decays at 700 and 1000 $\mathrm{nm}$ at different excitation intensities from 0.8 to $30 \mu \mathrm{J} \mathrm{cm}^{-2}$. Here the charge carrier density is calculated on the basis of the molar absorption coefficient of each polaron. As shown in the figure, both decays can be well fitted with an empirical power-law equation.

$$
n(t)=\frac{n_{0}}{(1+a t)^{\alpha}}
$$

The exponent $\alpha$ for the localized polaron band at $1000 \mathrm{~nm}$ is $\sim 0.5$, which is consistent with previous reports $[8,16,67,68]$. As mentioned above, this power-law decay with an exponent $\alpha<1$ is characteristic of bimolecular recombination of trapped carriers having an exponential tail of polaron trap states (trap-limited bimolecular recombination) $[31,69,70]$. On the other hand, the exponent $\alpha$ for the delocalized polaron band at 700 $\mathrm{nm}$ is as high as unity, suggesting trap-free bimolecular recombination. 
The diffusion-limited bimolecular charge recombination dynamics is given by

$$
\frac{\mathrm{d} n(t)}{\mathrm{d} t}=-\gamma(t) n^{2}(t)
$$

where $n(t)$ is the carrier density and $\gamma(t)$ is the bimolecular recombination rate at a delay time $t$. Therefore, the bimolecular recombination rate can be expressed as a function of time by substituting eq 1 into eq 2

$$
\gamma(t)=-\frac{\mathrm{d} n(t)}{\mathrm{d} t} \frac{1}{n^{2}(t)}=\frac{a \alpha}{n_{0}}(1+a t)^{\alpha-1}
$$

Similarly, the bimolecular recombination rate can be also expressed as a function of the carrier density by substituting eq 1 into eq 3 .

$$
\gamma(n)=\frac{a \alpha}{n}\left(\frac{n}{n_{0}}\right)^{\frac{1}{\alpha}}
$$

As mentioned above, $\alpha$ is equal to $\sim 0.5$ for the localized polaron band at $1000 \mathrm{~nm}$ and unity for the delocalized polaron band at $700 \mathrm{~nm}$. Therefore, as shown in Figure 20, the bimolecular recombination rate is time-dependent $\gamma(t)$ for the localized polaron band at $1000 \mathrm{~nm}$, which decreases from $10^{-12}$ to $10^{-13} \mathrm{~cm}^{3} \mathrm{~s}^{-1}$ over the time range from $10^{-6}$ to $10^{-3} \mathrm{~s}$, while it is time-independent $\gamma=a n_{0}^{-1} \approx 10^{-12} \mathrm{~cm}^{3} \mathrm{~s}^{-1}$ for the delocalized polaron band at $700 \mathrm{~nm}$. Figure 21 shows the temperature dependence of the bimolecular recombination rate of (a) localized polarons at $1000 \mathrm{~nm}$ and (b) delocalized polarons observed at $700 \mathrm{~nm}$ over the carrier density range from $10^{16}$ to $10^{17} \mathrm{~cm}^{-3}$. For the localized polarons, the activation energy estimated from the slope in the Arrhenius plots is as large as 0.097 to $0.178 \mathrm{eV}$, which depends on the carrier density. On the other hand, the activation energy for the delocalized polarons is as low as $\sim 0.078 \mathrm{eV}$ 
and independent of the carrier density. We therefore assign the localized polarons to trapped polarons and the delocalized polarons to trap-free polarons.

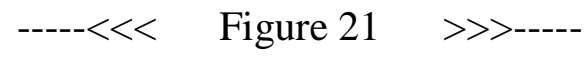

For localized polarons, the time-dependent trap-limited bimolecular recombination rate $\gamma(t)$ varies from $10^{-12}$ to $10^{-13} \mathrm{~cm}^{3} \mathrm{~s}^{-1}$ depending on time or carrier density, as shown in Figure 20, which is consistent with previous reports [67,68,71,72]. The time-dependent bimolecular recombination rate is due to trap depths depending on the carrier density: the trap depth deepens with time because of lower carrier density, resulting in the slower bimolecular recombination rate on longer time scales $[31,68,70]$. As shown in Figure 20b, the slope of $\log -\log$ plots of $\gamma(n)$ against $n$ is almost unity. In other words, $\gamma(n)$ can be expressed by $\gamma(n) \approx \gamma_{0} n$. Thus, eq 2 is rewritten as

$$
\frac{\mathrm{d} n(t)}{\mathrm{d} t} \approx-\gamma_{0} n^{3}(t)
$$

This is consistent with the trimolecular recombination dynamics reported recently for RR-P3HT:PCBM blends [67,68,71-73]. More specifically, $\gamma(n)$ can be expressed by $\gamma(n) \propto n^{(1 / \alpha)-1}$ where the exponent $(1 / \alpha)-1$ would vary from 4.3 to 2.4 because $\alpha$ varies from 0.3 to 0.7 depending on PCBM fractions or annealing conditions as reported previously $[8,16,67,68]$. In other words, the time-dependent trap-limited bimolecular recombination rate is strongly dependent on the film morphology.

For delocalized polarons, on the other hand, the time-independent trap-free bimolecular recombination rate is $\gamma \approx 10^{-12} \mathrm{~cm}^{3} \mathrm{~s}^{-1}$, which is one order of magnitude higher than that estimated by photo-CELIV [74]. This is probably because the 
photo-CELIV measurement cannot distinguish these two polarons and hence gives the averaged bimolecular recombination rate. Interestingly, the trap-free bimolecular recombination rate is several orders of magnitude lower than the Langevin recombination rate given by $\gamma_{\mathrm{L}}=e\left(\mu_{\mathrm{e}}+\mu_{\mathrm{h}}\right) / \varepsilon \varepsilon_{0}$ where $\mu_{\mathrm{e}}$ and $\mu_{\mathrm{h}}$ are the electron and hole mobility, respectively, and $\varepsilon$ and $\varepsilon_{0}$ are the relative permittivity of the film and the vacuum permittivity, respectively. Assuming $\mu_{\mathrm{e}} \approx 10^{-2} \mathrm{~cm}^{2} \mathrm{~V}^{-1} \mathrm{~s}^{-1}[75]$ and $\mu_{\mathrm{h}} \approx 10^{-4}$ $\mathrm{cm}^{2} \mathrm{~V}^{-1} \mathrm{~s}^{-1}$ [76], $\gamma_{\mathrm{L}}$ is estimated to be as high as $\sim 10^{-8} \mathrm{~cm}^{3} \mathrm{~s}^{-1}$. Considering the slowest mobility $\mu_{\mathrm{h}} \approx 10^{-4} \mathrm{~cm}^{2} \mathrm{~V}^{-1} \mathrm{~s}^{-1}$ [77], $\gamma_{\mathrm{L}}$ is estimated to be $\sim 10^{-10} \mathrm{~cm}^{3} \mathrm{~s}^{-1}$, which is still two orders of magnitude higher than the time-independent trap-free bimolecular recombination rate $\gamma \approx 10^{-12} \mathrm{~cm}^{3} \mathrm{~s}^{-1}$. As reported previously, the reduced bimolecular recombination rate is partly ascribed to phase-separated bicontinuous networks of RR-P3HT and PCBM domains, which are beneficial for reducing bimolecular recombination loss [67,74]. It is also possible that the recombination is not diffusion-limited but depends on the electron transfer rate at the interface. More importantly, the lifetime of trap-free carriers is estimated to be $\tau=\left(\gamma n_{0}\right)^{-1} \approx 10 \mu$ s under the 1 sun condition, which is longer than a charge collection time ( $2 \mu \mathrm{s})$ to extract $\sim 50 \%$ charges under the 1 sun open-circuit conditions [25]. This finding suggests that the majority of trap-free charge carriers could reach the electrode before the bimolecular recombination even under near open-circuit condition. Under the short-circuit condition, the recombination loss has been reported to be negligible because the short-circuit current increases linearly with the illumination intensity. We therefore conclude that trap-free polarons play a major role in the charge transport, resulting in the recombination-lossless performance in RR-P3HT:PCBM solar cells under not only the short-circuit but also near-open-circuit condition. This is consistent with the 
relatively high fill factors (0.6-0.7) and EQEs (>80\%) reported for this device in comparison with other combination devices [5-9].

\subsection{Relevance to Device Performance}

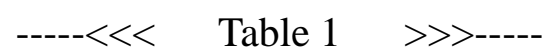

As summarized in Table 1, we can evaluate all the efficiency of photovoltaic events in polymer solar cells. As described above, we can evaluate the efficiency of $\eta_{\mathrm{ED}}, \eta_{\mathrm{CT}}$, and $\eta_{\mathrm{CD}}$ from the transient absorption study. The remaining charge collection efficiency $\eta_{\text {CC }}$ can be estimated from the internal quantum efficiency (IQE) reported in previous studies [57,78-80]. Note that the efficiency is not absolute one but should depend on the film morphology. Indeed, the difference in the IQE is due to the different film morphology depending on the preparation conditions. Nonetheless, the efficiency listed in the table demonstrates which loss process is dominant in the device performance qualitatively. For RR-P3HT:PCBM solar cells, all the efficiency is more than $90 \%$ after the thermal annealing. For RRa-P3HT:PCBM solar cells, on the other hand, the low efficiency in $\eta_{\mathrm{CD}}$ and $\eta_{\mathrm{CC}}$ is a major cause of the poor device performance. More specifically, the exciton diffusion $\eta_{\mathrm{ED}}$ is $\sim 100 \%$ for RRa-P3HT:PCBM blend films, 93\% for RR-P3HT:PCBM blend films before the thermal annealing, and 89\% after the thermal annealing. In terms of the exciton collection to the interface, therefore, homogeneously-mixed blend structures of RRa-P3HT:PCBM films are more desirable than phase-separated blend structures of 
RR-P3HT:PCBM films. This is consistent with the PL quenching results, indicating that there is still room to further improve the exciton diffusion efficiency in RR-P3HT:PCBM [81]. Indeed, such unquenched P3HT excitons can be effectively collected to the interface through the long-range energy transfer by loading appropriate dye molecules into RR-P3HT:PCBM blends [78,82]. For the charge transfer at the interface, $\eta_{\mathrm{CT}}$ is as high as $\sim 100 \%$ both for RRa-P3HT:PCBM and RR-P3HT:PCBM blend films, suggesting that it is dependent on the combination of donor and acceptor materials rather than blend structures. For the charge dissociation, $\eta_{\mathrm{CD}}$ is as low as 30\% for RRa-P3HT:PCBM blend films, while it is as high as $80 \%$ for RR-P3HT:PCBM blend films before the thermal annealing and is improved to 93\% after the thermal annealing. For the charge collection, $\eta_{\mathrm{CC}}$ is as low as $15 \%$ for RRa-P3HT:PCBM blend films, while it is $~ 60-70 \%$ for RR-P3HT:PCBM blend films before the thermal annealing and is improved up to $>90 \%$ after the thermal annealing. The large differences in $\eta_{\mathrm{CD}}$ and $\eta_{\mathrm{CC}}$ are mainly ascribed to the phase-separated networks and the crystallization of RR-P3HT, both of which result in improved carrier mobility and larger separation of bound radical pairs. The high charge collection efficiency is consistent with our conclusion in the previous section. In conclusion, there is not much difference in the charge generation yield between RRa-P3HT:PCBM and RR-P3HT:PCBM blend films. Rather, the charge dissociation and collection have a critical impact on the device performance of P3HT:PCBM solar cells. Further studies are required to address the origin of high efficiency in the charge dissociation and collection in polymer solar cells. 


\section{Concluding Remarks}

Polymer solar cells have made rapid progress in the device performance during the last decade. In 2010, a PCE in excess of $8 \%$ has been listed in the solar cell efficiency tables. More than $10 \%$ efficiency is now within reach. For such remarkable progress, development of various new materials has played a leading role. On the other hand, there still remain unsolved issues on the underlying mechanism of photovoltaic conversion in polymer solar cells. The device performance based on $J-V$ characteristics just gives us the final result of a series of fundamental photovoltaic conversion events such as photon absorption, exciton generation, exciton diffusion, charge separation, charge recombination, charge dissociation, charge transport, and charge collection. These range over nine orders of magnitude on temporal scale from $10^{-14}$ to $10^{-5} \mathrm{~s}$. Here we demonstrated how powerful and useful the transient absorption spectroscopy is for directly observing transient species such as excitons and polarons involved in photovoltaic conversion events. A transient absorption study will help us understand "what" is going on in the device and evaluate quantitatively "how" fast and efficient each event is. Consequently, we can discuss in detail "why" such device performance is obtained on the basis of not the final result but each fundamental photovoltaic conversion event. Such an in-depth understanding of the mechanism underlying polymer solar cells will lead to the development of new materials and progress in device engineering. In particular, the key to further improvements of polymer solar cells is to understand the origin of high charge dissociation and collection efficiency. The high dissociation efficiency cannot be explained rationally by the classical theory, and the reduced bimolecular recombination rate is inconsistent with the Langevin recombination. Further progress toward 15\% efficiency requires the synergy 
between developments of new materials and device structures and better understanding of the photovoltaic conversion mechanism.

\section{Acknowledgments}

This work was partly supported by the Kansai Research Foundation for Technology Promotion, the JST PRESTO program (Photoenergy and Conversion Systems and Materials for the Next-Generation Solar Cells) and the Global COE program (International Center for Integrated Research and Advanced Education in Materials Science) from the Ministry of Education, Culture, Sports, Science, and Technology, Japan. 


\section{References}

[1] Shaheen SE, Ginley DS, Jabbour GE (2005) Organic-based photovoltaics: Toward low-cost power generation. MRS Bull 30:10-19.

[2] Brabec CJ, Durrant JR (2008) Solution-processed organic solar cells. MRS Bull 33:670-675.

[3] Krebs FC (2009) Fabrication and processing of polymer solar cells: A review of printing and coating techniques. Sol Energy Mater Sol Cells 93:394-412.

[4] Padinger F, Rittberger RS, Sariciftci NS (2003) Effects of postproduction treatment on plastic solar cells. Adv Funct Mater 13:85-88.

[5] Li G, Shrotriya V, Huang J, Yao Y, Moriarty T, Emery K, Yang Y (2005) High-efficiency solution processable polymer photovoltaic cells by self-organization of polymer blends. Nat Mater 4:864-868.

[6] Ma W, Yang C, Gong X, Lee K, Heeger AJ (2005) Thermally stable, efficient polymer solar cells with nanoscale control of the interpenetrating network morphology. Adv Funct Mater 15:1617-1622.

[7] Kim JY, Kim SH, Lee HH, Lee K, Ma W, Gong X, Heeger AJ (2006) New architecture for high-efficiency polymer photovoltaic cells using solution-based titanium oxide as an optical spacer. Adv Mater 18:572-576.

[8] Kim Y, Cook S, Tuladhar SM, Choulis SA, Nelson J, Durrant JR, Bradley DDC, Giles M, McCulloch I, Ha CS, Ree M (2006) A strong regioregularity effect in self-organizing conjugated polymer films and high-efficiency polythiophene:fullerene solar cells. Nat Mater 5:197-203.

[9] Irwin MD, Buchholz DB, Hains AW, Chang RPH, Marks TJ (2008) p-Type 
semiconducting nickel oxide as an efficiency-enhancing anode interfacial layer in polymer bulk-heterojunction solar cells. Proc Natl Acad Sci USA $105: 2783-2787$.

[10] McCullough RD, Lowe RD (1992) Enhanced electrical conductivity in regioselectively synthesized poly(3-alkylthiophenes). J Chem Soc, Chem Commun 70-72.

[11] Chen TA, Rieke RD (1992) The first regioregular head-to-tail poly(3-hexylthiophene-2,5-diyl) and a regiorandom isopolymer: Ni vs Pd catalysis of 2(5)-bromo-5(2)-(bromozincio)-3-hexylthiophene polymerization. J Am Chem Soc 114:10087-10088.

[12] Chen TA, Wu X, Rieke RD (1995) Reigocontrolled synthesis of poly(3-alkylthiophenes) mediated by Rieke zinc: Their characterization and solid-state properties. J Am Chem Soc 117:233-244.

[13] Sirringhaus H, Brown PJ, Friend RH, Nielsen MM, Bechgaard K, Langeveld-Voss BMW, Spiering AJH, Janssen RAJ, Meijer EW, Herwig P, de Leeuw DM (1999) Two-dimensional charge transport in self-organized, high-mobility conjugated polymers. Nature 401:685-688.

[14] Green MA, Emery K, Hishikawa Y, Warta W (2011) Solar cell efficiency tables (version 37). Prog Photovolt: Res Appl 19:84-92.

[15] Service RF (2011) Outlook brightens for plastic solar cells. Science 332:293.

[16] Ohkita H, Cook S, Astuti Y, Duffy W, Tierney S, Zhang W, Heeney M, McCulloch I, Nelson J, Bradley DDC, Durrant JR (2008) Charge carrier formation in polythiophene/fullerene blend films studied by transient absorption spectroscopy. J Am Chem Soc 130:3030-3042. 
[17] Yamamoto S, Guo J, Ohkita H, Ito S (2008) Formation of methanofullerene cation in bulk heterojunction polymer solar cells studied by transient absorption spectroscopy. Adv Funct Mater 18:2555-2562.

[18] Guo J, Ohkita H, Benten H, Ito S (2009) Near-IR femtosecond transient absorption spectroscopy of ultrafast polaron and triplet exciton formation in polythiophene films with different regioregularities. J Am Chem Soc 131:16869-16880.

[19] Guo J, Ohkita H, Benten H, Ito S (2010) Charge generation and recombination dynamics in poly(3-hexylthiophene)/fullerene blend films with different regioregularities and morphologies. J Am Chem Soc 132:6154-6164.

[20] Guo J, Ohkita H, Benten H, Ito S (2010) Bimodal polarons and hole transport in poly(3-hexylthiophene):fullerene blend films. J Am Chem Soc 132:9631-9637.

[21] Ohkita H, Kosaka J, Guo J, Benten H, Ito S (2011) Charge generation dynamics in polymer/polymer solar cells studied by transient absorption spectroscopy. J Photon Energy 1:011118.

[22] Gregg BA (2004) Toward a unified treatment of electronic processes in organic semiconductors. J Phys Chem B 108:17285-17289.

[23] Pope M, Swenberg CE (1999) Electronic processes in organic crystals and polymers 2nd ed. Oxford University Press, New York.

[24] Brabec CJ, Zerza G, Cerullo G, Silvestri SD, Luzzati S, Hummelen JC, Sariciftci S (2001) Tracing photoinduced electron transfer process in conjugated polymer/fullerene bulk heterojunctions in real time. Chem Phys Lett 340:232-236. 
[25] Shuttle CG, Maurano A, Hamilton R, O’Regan B, de Mello JC, Durrant JR (2008) Charge extraction analysis of charge carrier densities in a polythiophene/fullerene solar cell: Analysis of the origin of the device dark current. Appl Phys Lett 93:183501.

[26] Westenhoff S, Howard IA, Hodgkiss JM, Kirov KR, Bronstein HA, Williams CK, Greenham NC, Friend RH (2008) Charge recombination in organic photovoltaic devices with high open-circuit voltages. J Am Chem Soc 130:13653-13658.

[27] Turro NJ (1978) Modern molecular photochemistry. The Benjamin/Cummings Publishing Company, Inc, California.

[28] Schweiger A (2003) Pulsed electron spin resonance spectroscopy: basic principles, techniques, and examples of applications. Angew Chem Int Ed 30:265-292.

[29] Österbacka R, An CP, Jiang XM, Vardeny ZV (2000) Two-dimensional electronic excitations in self-assembled conjugated polymer nanocrystals. Science 287:839-842.

[30] Jiang XM, Österbacka R, Korovyanko O, An CP, Horovitz B, Janssen RAJ, Vardeny ZV (2002) Spectroscopic studies of photoexcitations in regioregular and regiorandom polythiophene films. Adv Funct Mater 12:587-597.

[31] Nelson J (2003) Diffusion-limited recombination in polymer-fullerene blends and its influence on photocurrent collection. Phys Rev B 67:155209.

[32] Steigman J, Cronkright W (1970) Formation of Wurster's Blue in benzene at 25․ J Am Chem Soc 92:6736-6743.

[33] Guldi DM, Prato M (2000) Excited-state properties of $\mathrm{C}_{60}$ fullerene derivatives. 
Acc Chem Res 33:695-703.

[34] Kawaguchi T, Seki S, Okamoto K, Saeki Y, Yoshida Y, Tagawa S (2003) Pulse radiolysis study of radical cations of polysilanes. Chem Phys Lett 374:353-357.

[35] Mikhnenko OV, Cordella F, Sieval AB, Hummelen JC, Blom PWM, Loi MA (2008) Temperature dependence of exciton diffusion in conjugated polymers. J Phys Chem B 112:11602-11604.

[36] Shaw PE, Ruseckas A, Samuel IDW (2008) Exciton diffusion measurements in poly(3-hexylthiophene) Adv Mater 20:3516-3520.

[37] Cook S, Liyuan H, Furube A, Katoh R (2010) Singlet annihilation in films of regioregular poly(3-hexylthiophene): Estimates for singlet diffusion lengths and the correlation between singlet annihilation rates and spectral relaxation. $\mathrm{J}$ Phys Chem C 114:10962-10968.

[38] Kraabel B, Moses D, Heeger AJ (1995) Direct observation of the intersystem crossing in poly(3-octylthiophene). J Chem Phys 103:5102-5108.

[39] Sheng Y, Nguyen TD, Veeraraghavan G, Mermer Ö, Wohlgenannt M, Qiu S, Scherf U (2006) Hyperfine interaction and magnetoresistance in organic semiconductors. Phys Rev B 74:045213.

[40] Verhoeven JW (2006) On the role of spin correlation in the formation, decay, and detection of long-lived, intramolecular charge-transfer states. J Photochem Photobiol C: Photochem Rev 7:40-60.

[41] Smith MB, Michl J (2010) Singlet fission. Chem Rev 110:6891-6936.

[42] Singh S, Jones WJ, Siebrand W, Stoicheff BP, Schneider WG (1965) Laser generation of excitons and fluorescence in anthracene crystals. J Chem Phys 
42:330-342.

[43] Katoh R, Kotani M (1992) Fission of a higher excited state generated by singlet exciton fusion in an anthracene crystals. Chem Phys Lett 196:108-112.

[44] Merrifield RE, Avakian P, Groff RP (1969) Fission of singlet excitons into pairs of triplet excitons in tetracene crystals. Chem Phys Lett 3:386-388.

[45] Albrecht WG, Michel-Beyerle ME, Yakhot V (1978) Exciton fission in excimer forming crystal. Dynamics of an excimer build-up in $\alpha$-perylene. Chem Phys 35:193-200.

[46] Zenz C, Cerullo G, Lanzani G, Graupner W, Meghdadi F, Leising G, Silvestri SD (1999) Ultrafast photogeneration mechanisms of triplet states in para-hexylphenyl. Phys Rev B 59:14336-14341.

[47] Katoh R, Kotani M, Hirata Y, Okada T (1997) Triplet exciton formation in a benzophenone single crystal studied by picosecond time-resolved absorption spectroscopy. Chem Phys Lett 264:631-635.

[48] Österbacka R, Wohlgenannt M, Shkunov M, Chinn D, Vardeny ZV (2003) Excitons, polarons, and laser action in poly(p-phenylene vinylene) films. J Chem Phys 118:8905-8916.

[49] Wohlgenannt M, Graupner W, Leising G, Vardeny ZV (1999) Photogeneration action spectroscopy of neutral and charged excitations in films of a ladder-type poly(para-phenylene). Phys Rev Lett 82:3344-3347.

[50] Kraabel B, Hulin D, Aslangul C, Lapersonne-Meyer C, Schott M (1998) Triplet exciton generation, transport and relaxation in isolated polydiacetylene chains: Subpicosecond pump-probe experiments. Chem Phys 227:83-98.

[51] Köhler A, Wilson JS, Friend RH, Al-Suti MK, Khan MS, Gerhard A, Bässler H 
(2002) The singlet-triplet energy gap in organic and Pt-containing phenylene ethynylene polymers and monomers. J Chem Phys 116:9457-9463.

[52] Köhler A, Beljonne D (2004) The singlet-triplet exchange energy in conjugated polymers. Adv Funct Mater 14:11-18.

[53] Nozik AJ (2008) Multiple exciton generation in semiconductor quantum dots. Chem Phys Lett 457:3-11.

[54] Nozik AJ (2010) Nanoscience and nanostructures for photovoltaics and solar fuels. Nano Lett 10:2735-2741.

[55] Lee J, Jadhav P, Baldo MA (2009) High efficiency organic multilayer photodetectors based on singlet exciton fission. Appl Phys Lett 95:033301.

[56] Rao A, Wilson MWB, Hodgkiss JM, Albert-Seifried S, Bässler H, Friend RH (2010) Exciton fission and charge generation via triplet excitons in pentacene/C 60 bilayers. J Am Chem Soc 132:12698-12703.

[57] Marsh RA, Hodgkiss JM, Albert-Seifreid S, Friend RH (2010) Effect of annealing on P3HT:PCBM charge transfer and nanoscale morphology probed by ultrafast spectroscopy. Nano Lett 10:923-930.

[58] Howard IA, Mauer R, Meister M, Laquai F (2010) Effect of morphology on ultrafast free carrier generation in polythiophene:fullerene organic solar cells. J Am Chem Soc 132:14866-14876.

[59] Onsager L (1938) Initial recombination of ions. Phys Rev 54:554-557.

[60] Braun CL (1984) Electric field assisted dissociation of charge transfer states as a mechanism of photocarrier production. J Chem Phys 80:4157-4161.

[61] Peumans P, Forrest SR (2004) Separation of geminate charge-pairs at donor-acceptor interfaces in disordered solids. Chem Phys Lett 398:27-31. 
[62] Groves C, Marsh RA, Greenham NC (2008) Monte Carlo modeling of geminate recombination in polymer-polymer photovoltaic devices. J Chem Phys 129:114903.

[63] Wojcik M, Michalak P, Tachiya M (2010) Geminate electron-hole recombination in organic solids in the presence of a donor-acceptor heterojunction. Appl Phys Lett 96:162102.

[64] Clarke TM, Durrant JR (2011) Charge photogeneration in organic solar cells. Chem Rev 110:6736-6767.

[65] Deibel C, Strobel T (2009) Origin of the efficient polaron-pair dissociation in polymer-fullerene blends. Phys Rev Lett 103:036402.

[66] Hilczer M, Tachiya M (2010) Unified theory of geminate and bulk electron-hole recombination in organic solar cells. J Phys Chem 114:6808-6813.

[67] Shuttle CG, O’Regan B, Ballantyne AM, Nelson J, Bradley DDC, Durrant JR (2008) Bimolecular recombination losses in polythiophene:fullerene solar cells. Phys Rev B 78:113201.

[68] Clarke TM, Jamieson FC, Durrant JR (2009) Transient absorption studies of bimolecular recombination dynamics in polythiophene/fullerene blend films. J Phys Chem C 113:20934-20941.

[69] Montanari I, Nogueira AF, Nelson J, Durrant JR, Winder C, Loi MA, Sariciftci NS, Brabec CJ (2002) Transient optical studies of charge recombination dynamics in a polymer/fullerene composite at room temperature. Appl Phys Lett 81:3001-3003. 
[70] Nogueira AF, Montanari I, Nelson J, Durrant JR, Winder C, Sariciftci NS, Brabec C (2003) Charge recombination in conjugated polymer/fullerene blended films studied by transient absorption spectroscopy. J Phys Chem B 107:1567-1573.

[71] Shuttle CG, O’Regan B, Ballantyne AM, Nelson J, Bradley DDC, de Mello J, Durrant JR (2008) Experimental determination of the rate law for charge carrier density in a polythiophene:fullerene solar cell. Appl Phys Lett 92:093311.

[72] Foertig A, Baumann A, Rauh D, Dyakonov V, Deibel C (2009) Charge carrier concentration and temperature dependent recombination in polymer-fullerene solar cells. Appl Phys Lett 95:052104.

[73] Juška G, Genevičius K, Nekrašas N, Sliaužys G, Dennler G (2008) Trimolecular recombination in polythiophene:fullerene bulk heterojunction solar cells. Appl Phys Lett 93:143303.

[74] Pivrikas A, Juška G, Mozer A, Scharber M, Arlauskas K, Sariciftci NS, Stubb H, Österbacka R (2005) Bimolecular recombination coefficient as a sensitive testing parameter for low-mobility solar-cell materials. Phys Rev Lett 94:176806.

[75] Tuladhar SM, Poplavskyy D, Choulis SA, Durrant JR, Bradley DDC, Nelson J (2005) Ambipolar charge transport in films of methanofullerene and poly(phenylenevinylene)/methanofullerene blends. Adv Funct Mater 15:1171-1182. 
[76] Baumann A, Lorrmann J, Deibel C, Dyakonov V (2008) Bipolar charge transport in poly(3-hexyl thiophene)/methanofullerene blends: A ratio dependent study. Appl Phys Lett 93:252104.

[77] Koster LJA, Mihailetchi VD, Blom PWM (2006) Bimolecular recombination in polymer/fullerene bulk heterojunction solar cells. Appl Phys Lett 88:052104.

[78] Honda S, Nogami T, Ohkita H, Benten H, Ito S (2009) Improvement of the light-harvesting efficiency in polymer/fullerene bulk heterojunction solar cells by interfacial dye modification. ACS Appl Mater Interfaces 1:804-810.

[79] Campoy-Quiles M, Kanai Y, El-Basaty A, Sakai H, Murata H (2009) Ternary mixing: A simple method to tailor the morphology of organic solar cells. Org Electron 10:1120-1132.

[80] Takanezawa K, Tajima K, Hashimoto K Efficiency enhancement of polymer photovoltaic devices hybridized with $\mathrm{ZnO}$ nanorod arrays by the introduction of a vanadium oxide buffer. Appl Phys Lett 93:063308.

[81] Ayzner AL, Wanger DD, Tassone CJ, Tolbert SH, Schwartz BJ (2008) Room to improve conjugated polymer-based solar cells: Understanding how thermal annealing affects the fullerene component of a bulk heterojunction photovoltaic devices. J Phys Chem C 112:18711-18716.

[82] Honda S, Yokoya S, Ohkita H, Benten H, Ito S (2011) Light-harvesting mechanism in polymer/fullerene/dye ternary blends studied by transient absorption spectroscopy. J Phys Chem C 115:11306-11317. 


\section{Figure Captions}

Fig. 1. Photovoltaic conversion events in polymer solar cells with a bilayer structure of hole-transporting and electron-transporting materials: 1) exciton generation by photon absorption , 2) exciton diffusion into a donor/acceptor interface, 3) charge transfer at the interface, 4) charge dissociation into free carriers, and 5) charge transport to each electrode.

Fig. 2. Block diagram of highly sensitive microsecond transient absorption measurement system: MC monochromator, S sample, PC computer, and PD PIN photodiode to detect a part of a pump laser pulse as a trigger signal, which is sent to the digital oscilloscope. The detector is replaceable: Si PIN photodiode for the visible wavelength range and InGaAs PIN photodiode for the near-IR wavelength range. The black and gray lines represent electric signals and optical probe and pump light, respectively.

Fig. 3. Block diagram of pump and probe femtosecond transient absorption measurement system: SHG second harmonic generator, ODL optical delay line, C chopper, WLG white light generator, S sample, D detector. The detector is replaceable: a linear CCD array for the visible wavelength range and a digital line scan InGaAs camera for near-IR wavelength range.

Fig. 4. a) Transient absorption spectra of RRa-P3HT films excited at $400 \mathrm{~nm}$ measured at $0,1,10,100$, and 3000 ps from top to bottom. b) Transient absorption 
spectra of RRa-P3HT films excited at $450 \mathrm{~nm}$ measured at 0.5, 2, 4, 6, and $10 \mu$ s from top to bottom. The inset shows the transient absorption decay at $850 \mathrm{~nm}$ under Ar and $\mathrm{O}_{2}$ atmosphere.[18]

Fig. 5. a) Transient absorption spectra of RR-P3HT films measured at 0, 1, 10, 100, and 3000 ps from top to bottom in each panel. The excitation intensity is as follows: a) 15 , b) 30, c) 60, and d) $120 \mu \mathrm{J} \mathrm{cm}^{-2}$. The excitation wavelength is $400 \mathrm{~nm}$.[18]

Fig. 6. a) Transient absorption spectra of RRa-P3HT:PCBM (50:50 w/w) blend films (solid lines) measured at $0,0.2,1,100$, and 3000 ps (from top to bottom). The broken line represents transient absorption spectrum of an RRa-P3HT pristine film measured at 0 ps. The transient absorption is corrected for variation in the absorption at an excitation wavelength of $400 \mathrm{~nm}$. b) Transient absorption spectra of RRa-P3HT:PCBM (50:50 w/w) blend films excited at $450 \mathrm{~nm}$ measured at 0.5, 1, 2, 4, and $8 \mu$ s (from top to bottom). The inset shows transient absorption decays at 850 (upper) and $1030 \mathrm{~nm}$ (lower). The white broken lines represent fitting curves with a power-law equation: $\Delta \mathrm{OD}(t) \propto t^{-\alpha}$.[19]

Fig. 7. a) Transient absorption spectra of RR-P3HT:PCBM (50:50 w/w) blend films after thermal annealing (solid lines) measured at 0, 1, 10, 100, and 3000 ps (from top to bottom). The broken line represents transient absorption spectrum of an RR-P3HT pristine film (broken line) measured at 0 ps. The transient absorption is corrected for variation in the absorption at an excitation wavelength of $400 \mathrm{~nm}$. b) Transient absorption spectra of RR-P3HT:PCBM (50:50 w/w) blend films after thermal annealing 
excited at $400 \mathrm{~nm}$ measured at $0.5,1,2,5,10,20$ and $100 \mu$ s (from top to bottom). The inset shows transient absorption decays at 1000 (upper) and $700 \mathrm{~nm}$ (lower).[19,20]

Fig. 8. a) Transient absorption spectra of $\mathrm{PT}_{10} \mathrm{PhT}_{10}: \mathrm{PCBM}(95: 5 \mathrm{w} / \mathrm{w})$ blend films excited at $420 \mathrm{~nm}$ measured at $0,1,10,100$, and 3000 ps (from top to bottom). b) Transient absorption decays of $\mathrm{PT}_{10} \mathrm{PhT}_{10}: \mathrm{PCBM}(95: 5 \mathrm{w} / \mathrm{w})$ blend films excited at 420 $\mathrm{nm}$ monitored at $700 \mathrm{~nm}$ under argon (black line) and oxygen (gray line) atmosphere.[16]

Fig. 9. Transient absorption spectra of a polystyrene film doped with TMPD(20 wt\%) and PCBM(30 wt\%) at 1, 2, and 10 ps after the laser excitation at $400 \mathrm{~nm}$. The inset shows the transient decays at $600 \mathrm{~nm}$ (solid line) and $1050 \mathrm{~nm}$ (broken line). Reproduced with permission from [17]. Copyright Wiley-VCH Verlag GmbH \& Co. KGaA.

Fig. 10. Transient absorption spectra of P3HTpristine films measured at $0,1,10,100$, and 3000 ps from top to bottom in each panel: a) RRa-P3HT excited at $400 \mathrm{~nm}, \mathrm{~b})$ RR-P3HT excited at $400 \mathrm{~nm}$, and c) RR-P3HT excited at $620 \mathrm{~nm} .[18]$

Fig. 11. a) Transient absorption spectra of RR-P3HT:F8BT blend films excited at 400 nm measured at $0,1,10,100,1000$ ps from top to bottom. b) Time evolution of F8BT singlet excitons (open circles) and RR-P3HT singlet excitons (closed circles). The rise and decay curves are fitted with the following equation: $\Delta \mathrm{OD}_{\mathrm{R}}(t)=\mathrm{A}\left[1-\exp \left(-t / \tau_{\mathrm{R}}\right)\right]+$ 
$\mathrm{B}$ and $\Delta \mathrm{OD}_{\mathrm{D}}(t)=\mathrm{A} \exp \left(-t / \tau_{\mathrm{D}}\right)\left(\tau_{\mathrm{R}}=\tau_{\mathrm{D}}=0.3 \mathrm{ps}\right) \cdot[21]$

Fig. 12. Normalized transient absorption decays of RRa-P3HT pristine films excited at $400 \mathrm{~nm}\left(\sim 30 \mu \mathrm{J} \mathrm{cm}^{-2}\right)$. The closed circles represent time evolution of singlet excitons measured at $1000 \mathrm{~nm}$. The open circles represent time evolution of triplet excitons, which is evaluated by subtracting the transient signals of singlet excitons at $1000 \mathrm{~nm}$ from that at $825 \mathrm{~nm} .[18]$

Fig. 13. a) Normalized transient absorption signals of singlet exciton (closed circles) and polaron (closed triangles) generated in RRa-P3HT:PCBM (50:50 w/w) blend films excited at $400 \mathrm{~nm}$. The closed circles are obtained by subtracting the transient signal of polaron at $1600 \mathrm{~nm}$ (closed triangles) from that at $1000 \mathrm{~nm}$ (solid line, singlet exciton and polaron). The subtracted signals (closed circles) are fitted with a monoexponential function: $\Delta \mathrm{OD}(t)=\mathrm{A} \exp \left(-t / \tau_{\mathrm{D}}\right) . \quad$ The transient rise signals at $1600 \mathrm{~nm}$ are fitted with an exponential function and a constant: $\Delta \mathrm{OD}(t)=\mathrm{A}\left[1-\exp \left(-t / \tau_{\mathrm{R}}\right)\right]+\mathrm{B}\left(\tau_{\mathrm{D}}=\tau_{\mathrm{R}}=0.2\right.$ ps). b) Normalized transient absorption signals of photobleaching at $470 \mathrm{~nm}$ (closed squares) for RRa-P3HT:PCBM (50:50 w/w) blend films excited at $400 \mathrm{~nm}$. The photobleaching signals are fitted with a constant: $\Delta \mathrm{OD}(t)=$ constant. The white broken lines represent the best-fitting curves. The dotted line indicates the instrument response function of the transient absorption spectroscope.[19]

Fig. 14. Normalized transient absorption signals of RR-P3HT:PCBM blend films excited at $400 \mathrm{~nm}$ measured at $1200 \mathrm{~nm}$ (singlet excitons, closed circles) and $720 \mathrm{~nm}$ (polarons, open circles): a) $5 \mathrm{wt} \%$ PCBM, b) $20 \mathrm{wt} \%$ PCBM, c) 50wt\% PCBM before 
thermal annealing, and d) $50 \mathrm{wt} \%$ PCBM after thermal annealing. The transient absorption decay at $1200 \mathrm{~nm}$ is fitted with a double exponential function: $\Delta \mathrm{OD}(t)=$ $A_{D 1} \exp \left(-t / \tau_{\mathrm{D} 1}\right)+A_{\mathrm{D} 2} \exp \left(-t / \tau_{\mathrm{D} 2}\right) . \quad$ The transient absorption decay at $720 \mathrm{~nm}$ is fitted with a double exponential function: $\Delta \mathrm{OD}(t)=\mathrm{A}_{\mathrm{R}}\left[1-\exp \left(-t / \tau_{\mathrm{R}}\right)\right]+\mathrm{B}$. The gray lines represent the best-fitting curves.[19]

Fig. 15. Intensity dependence of the simulated transient absorption decays for a) the monomolecular recombination (the first order reaction) and b) the bimolecular recombination (the second order reaction). In the first order reaction, the lifetime is independent of the initial concentration $n_{0}$. In the second order reaction, the half-life is dependent on $n_{0}$ : it is half at twice the initial concentration.

Fig. 16. Normalized transient absorption decays of RRa-P3HT:PCBM (50:50 w/w) blend films excited at $400 \mathrm{~nm}$ measured at a) $1030 \mathrm{~nm}$ and b) $480 \mathrm{~nm}$. The transient decay is fitted with an exponential function and a constant: $\Delta \mathrm{OD}(t)=\mathrm{A}_{\mathrm{D}}\left[1-\exp \left(-t / \tau_{\mathrm{D}}\right)\right]$

+ B. The broken lines represent the best-fitting curve.[19]

Fig. 17. Transient absorption decays of RRa-P3HT:PCBM (50:50 w/w) blend films excited at $400 \mathrm{~nm}$ measured at a) $1030 \mathrm{~nm}$, b) $850 \mathrm{~nm}$, and c) $480 \mathrm{~nm}$. The excitation intensity is varied over $6,12,24,48,72,120 \mu \mathrm{J} \mathrm{cm}^{-2}$ from bottom to top in each panel. The negative signals at $480 \mathrm{~nm}$ are converted to positive.[19]

Fig. 18. Transient absorption decays of RR-P3HT:PCBM (50:50 w/w) blend films after thermal annealing excited at $400 \mathrm{~nm}$ measured at a) $700 \mathrm{~nm}$ and b) $1000 \mathrm{~nm}$. The 
excitation intensity is $6,12,24,48,72,120 \mu \mathrm{J} \mathrm{cm}^{-2}$ from bottom to top in each panel.[19]

Fig. 19. Time evolution of the carrier density $n(t)$ in RR-P3HT:PCBM (50:50 w/w) blend films measured at a) $700 \mathrm{~nm}$ and b) $1000 \mathrm{~nm}$. The excitation intensity at $400 \mathrm{~nm}$ is $0.8,1.8$, and $4.7 \mu \mathrm{J} \mathrm{cm}^{-2}$ from bottom to top in each panel. The broken lines represent fitting curves with an empirical power equation: $n(t)=n_{0} /(1+a t)^{-\alpha} \cdot[20]$

Fig. 20. Log-log plots of the bimolecular recombination rate $\gamma$ at $1000 \mathrm{~nm}$ (solid lines) and $700 \mathrm{~nm}$ (broken lines) as a function of a) time $t$ and b) the carrier density n.[20]

Fig. 21. Arrhenius plots of the bimolecular recombination rate $\gamma$ at a) $1000 \mathrm{~nm}$ and b) $700 \mathrm{~nm}$ over the temperature range from 150 to $290 \mathrm{~K}$. The carrier density $n$ is $1.3 \times$ $10^{16}(\diamond), 2.7 \times 10^{16}(\diamond), 5.4 \times 10^{16}(\bullet), 1.1 \times 10^{17}(\square), 1.6 \times 10^{17}(\bullet)$, and $2.2 \times 10^{17} \mathrm{~cm}^{-3}$ (○) at $700 \mathrm{~nm}$ and $1.3 \times 10^{16}(\diamond), 2.5 \times 10^{16}(\diamond), 5.0 \times 10^{16}(\mathbf{\square}), 1.0 \times 10^{17}(\square), 1.5 \times 10^{17}$ $(\bullet)$, and $2.0 \times 10^{17} \mathrm{~cm}^{-3}(\circ)$ at $1000 \mathrm{~nm}$ from bottom to top in each panel. The solid lines represent fitting curves with the Arrhenius equation: $\ln \gamma=\ln \mathrm{A}-E_{\mathrm{A}} /\left(k_{\mathrm{B}} T\right)$.[20] 


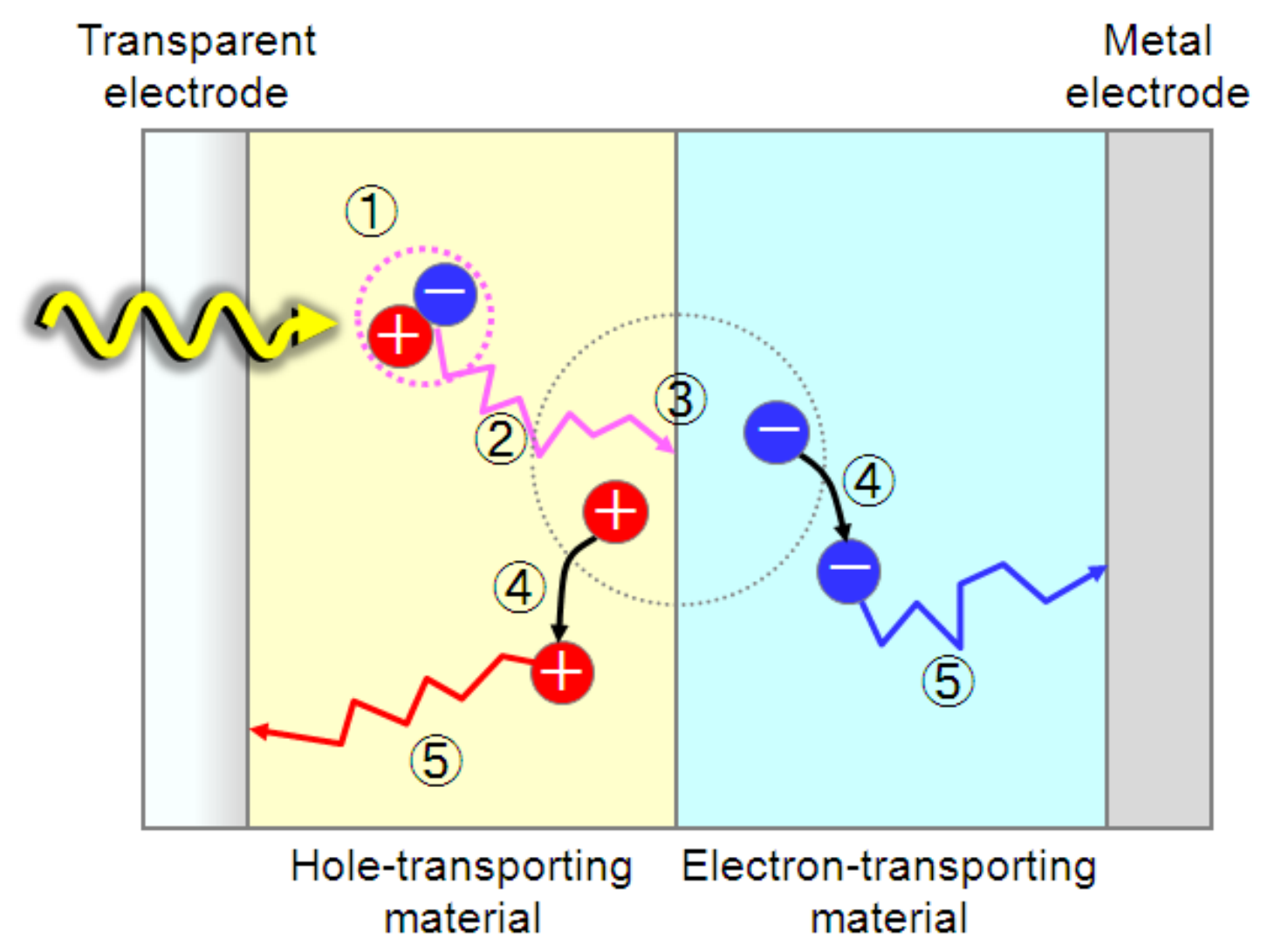

Fig. 1. 


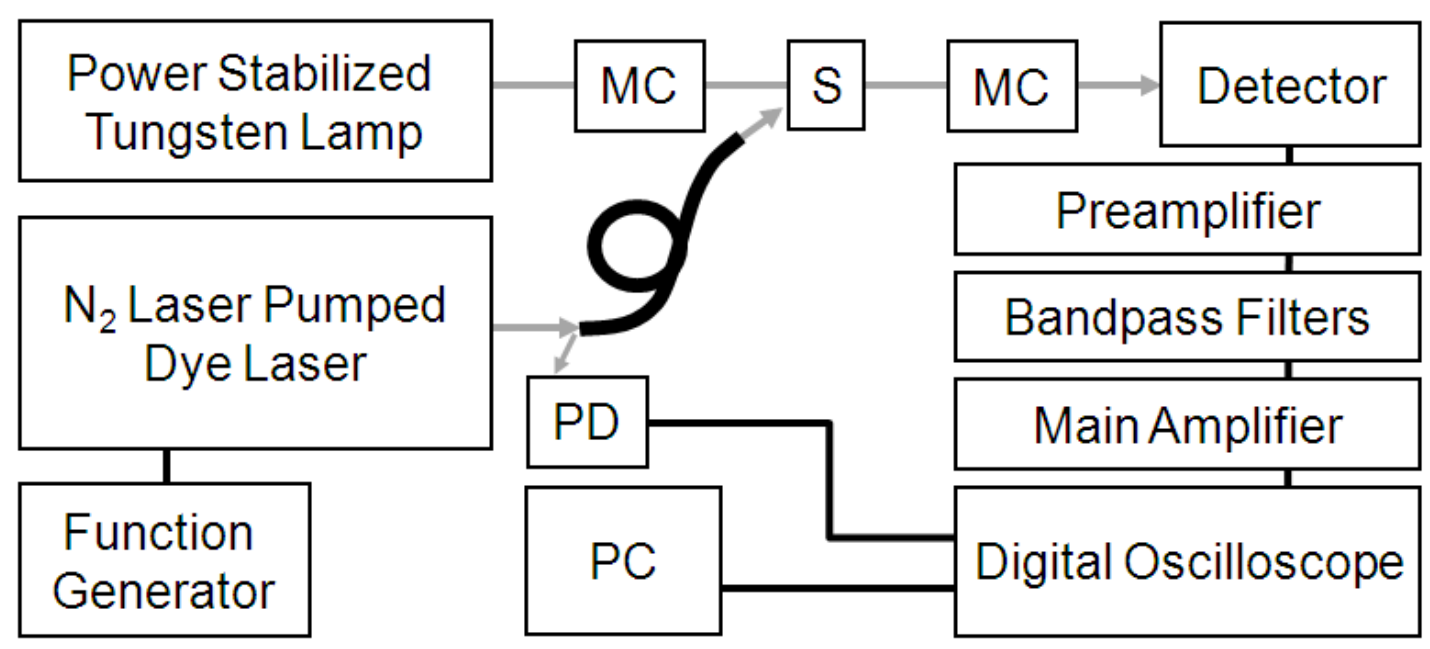

Fig. 2. 


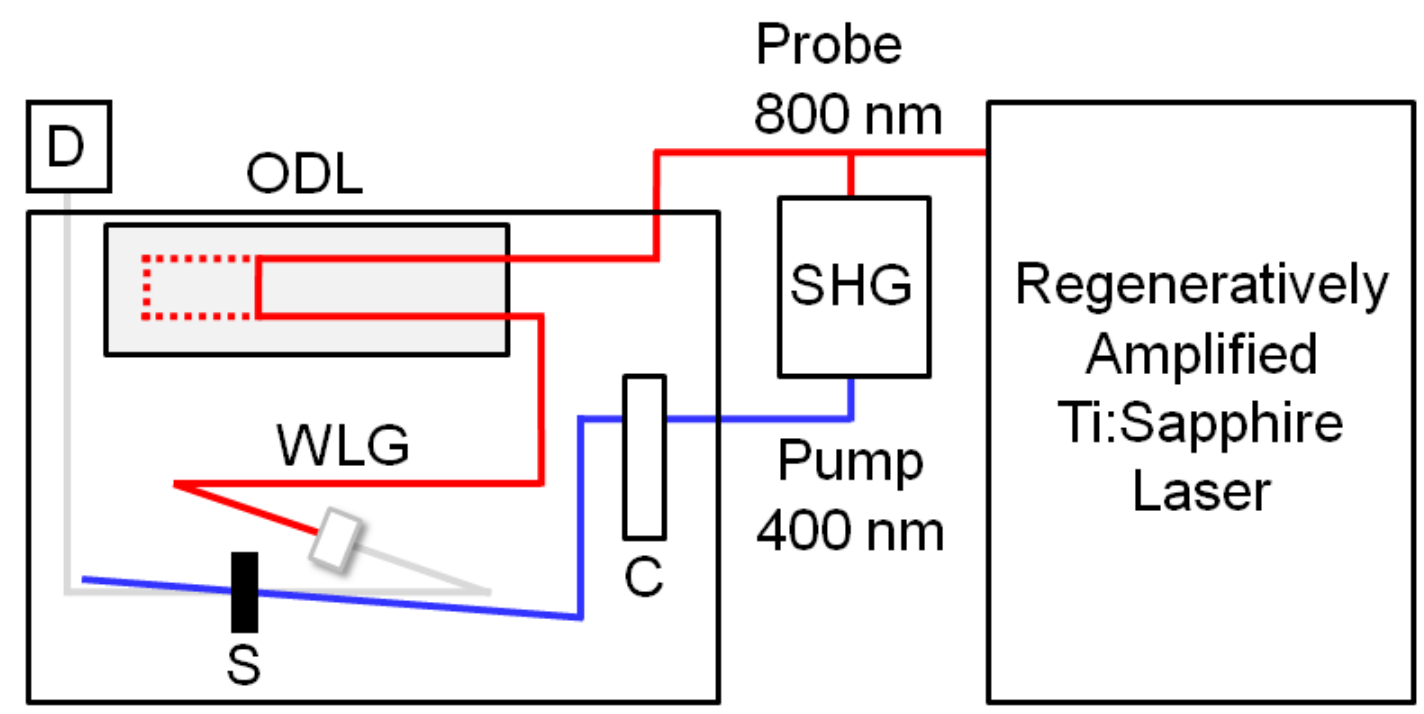

Fig. 3. 


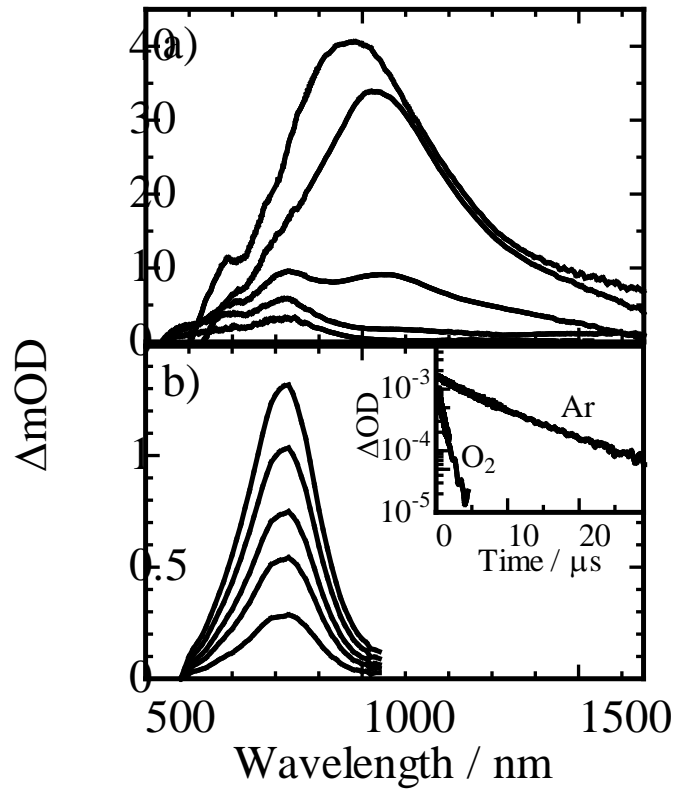

Fig. 4. 


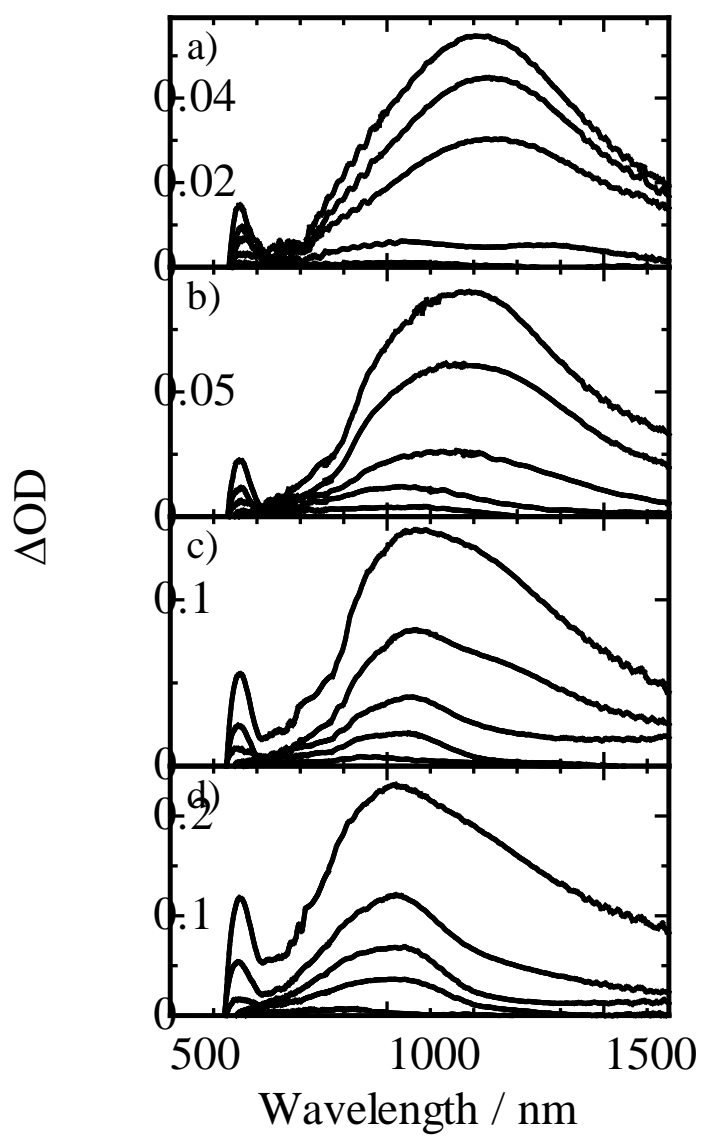

Fig. 5. 


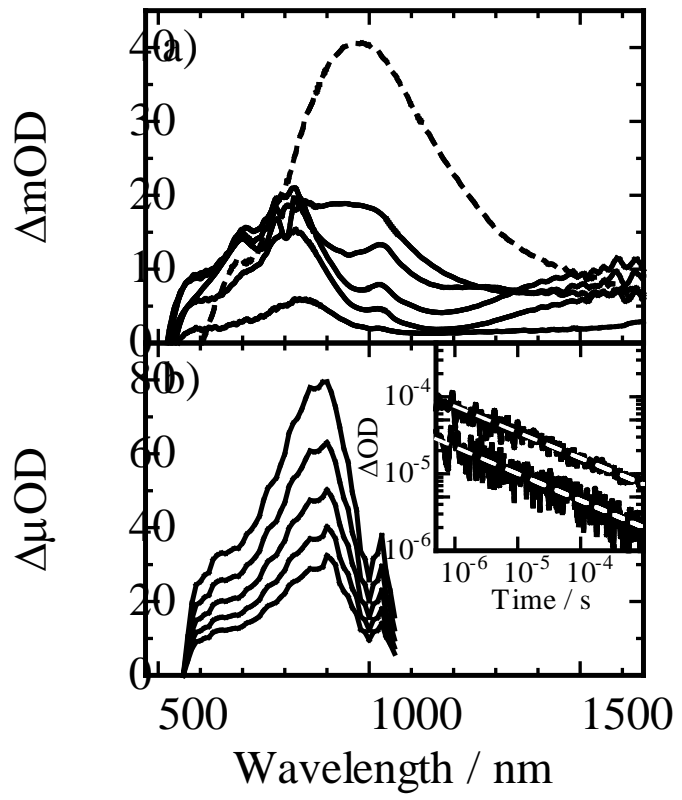

Fig. 6. 


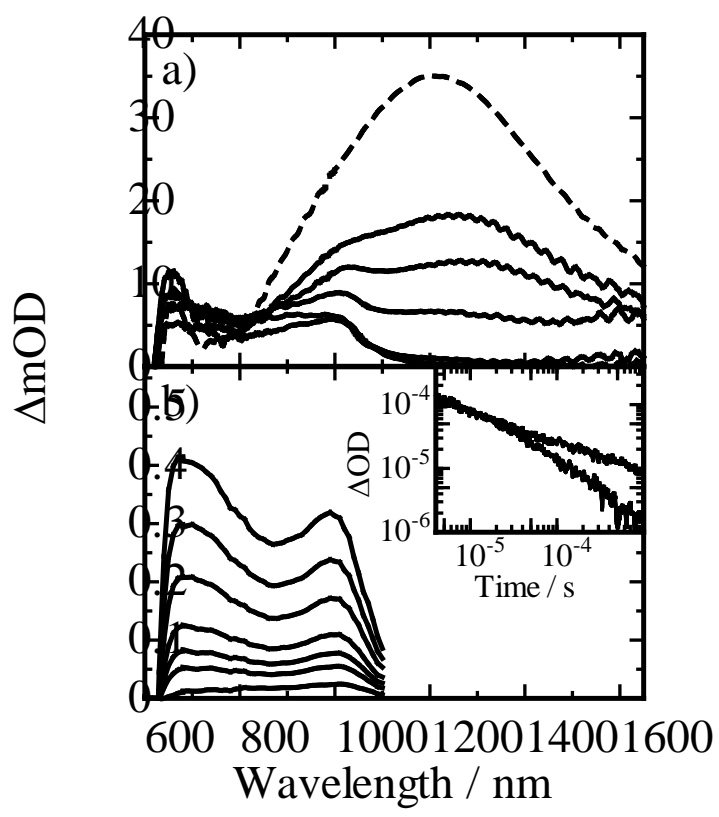

Fig. 7. 

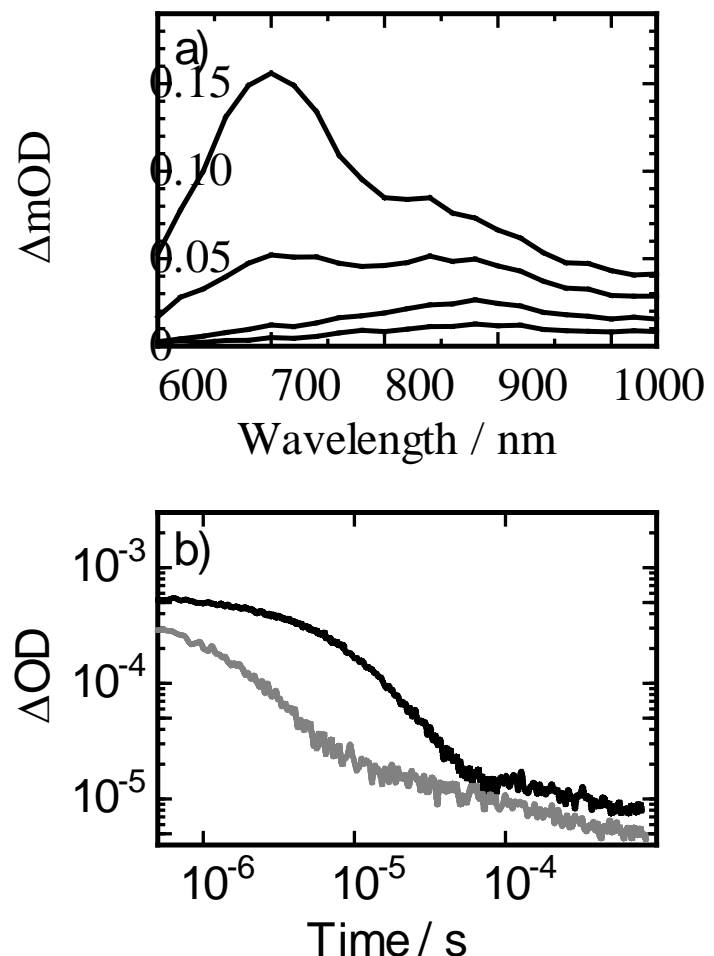

Fig. 8. 


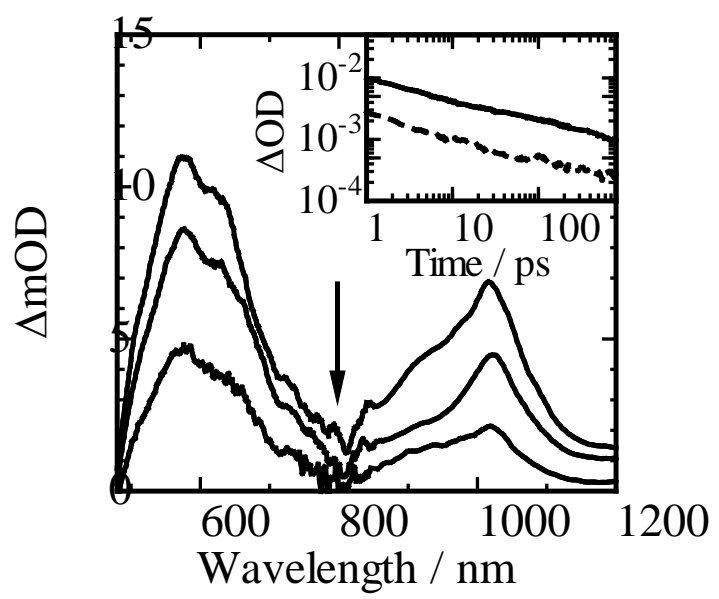

Fig. 9 


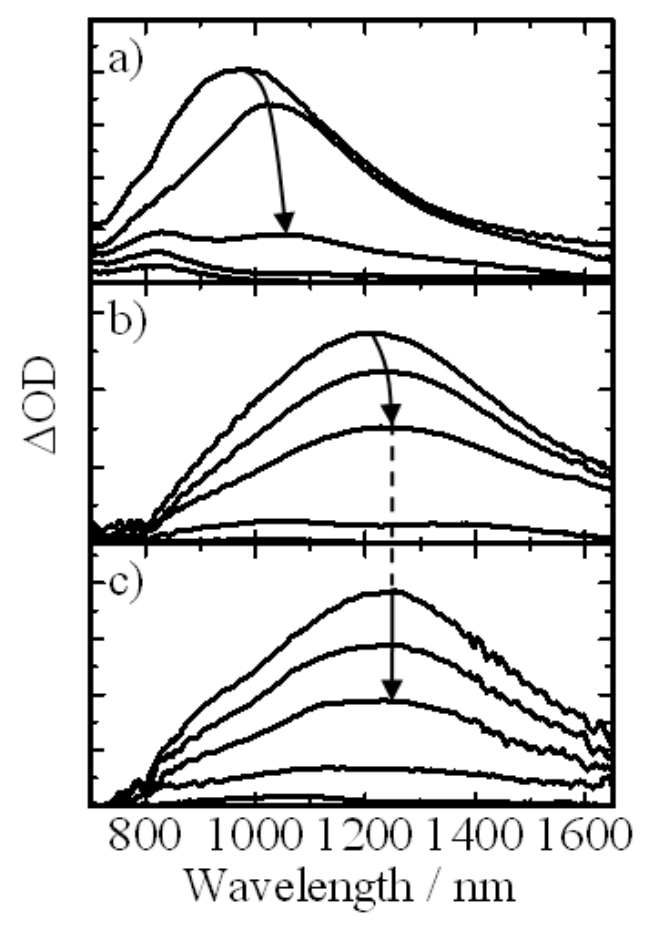

Fig. 10 

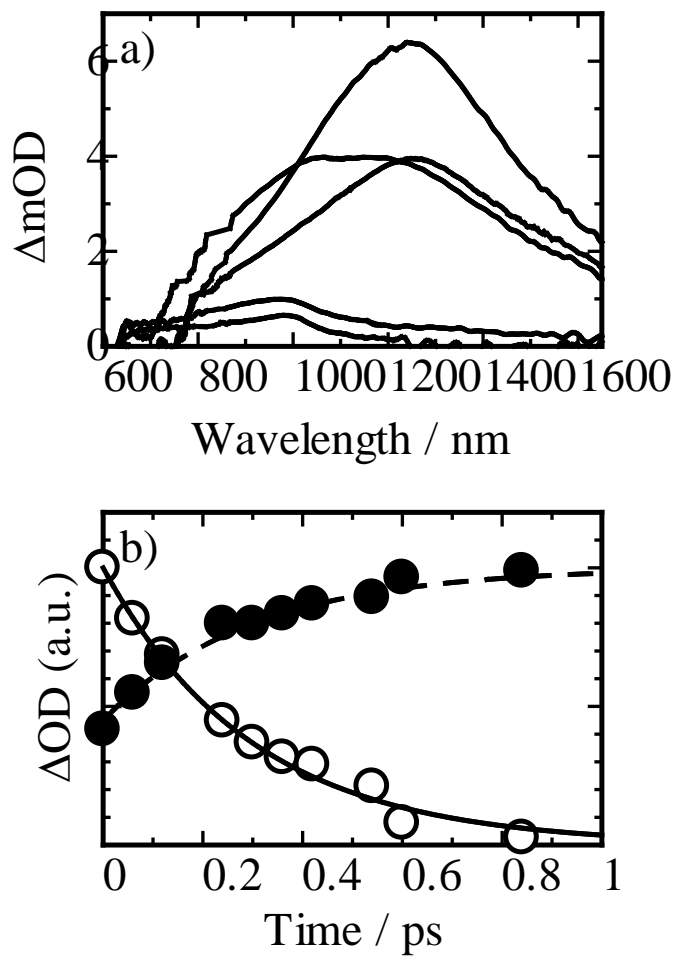

Fig. 11 


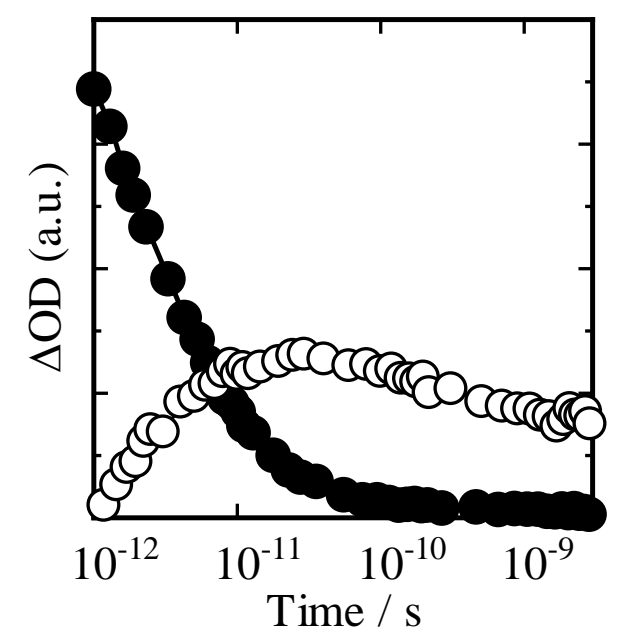

Fig. 12 


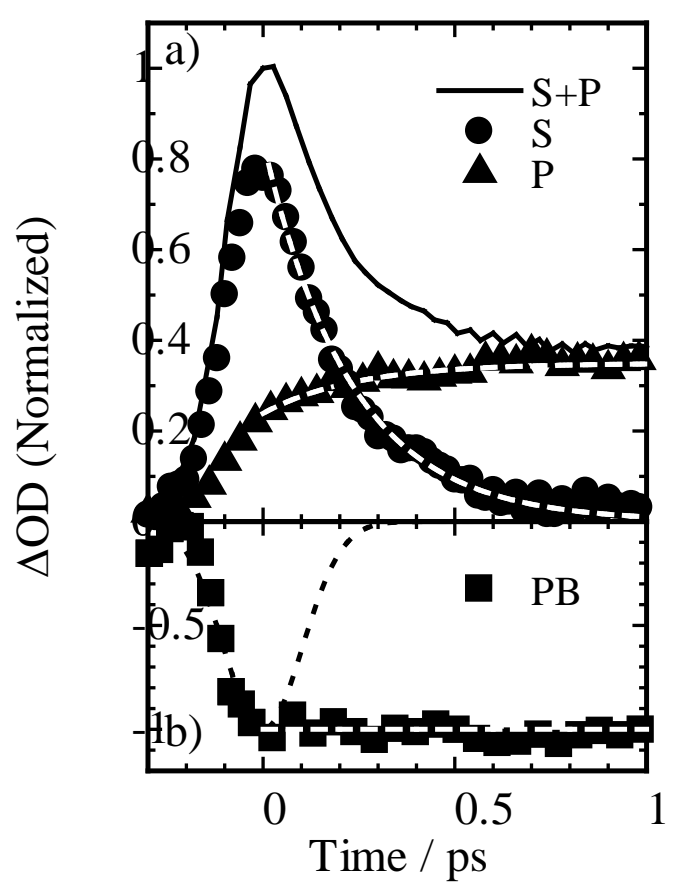

Fig. 13 


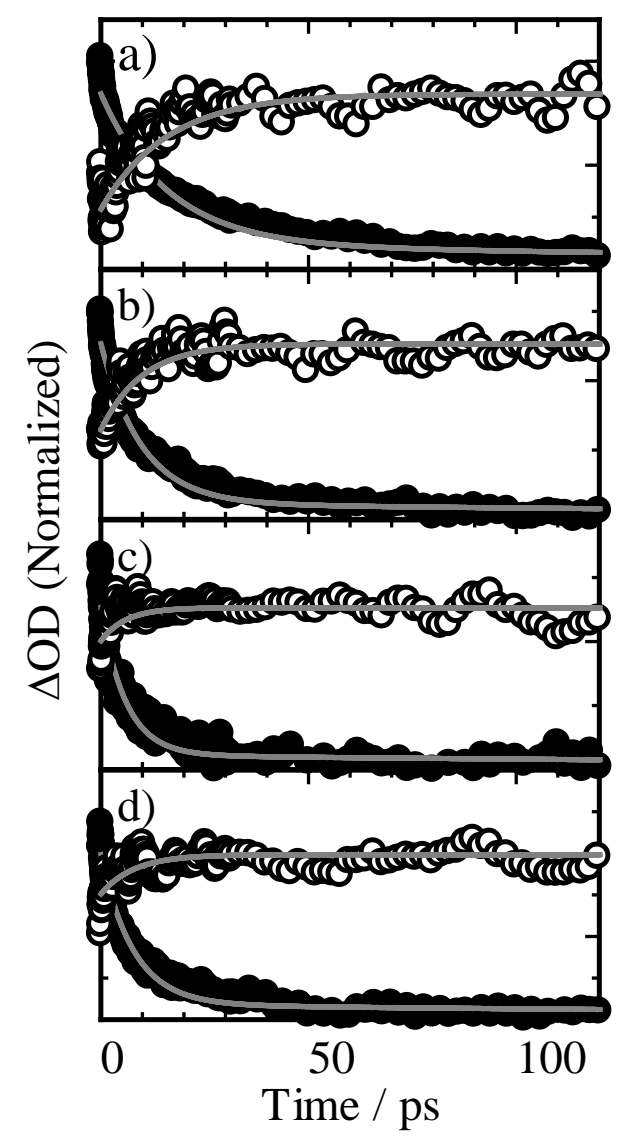

Fig. 14 


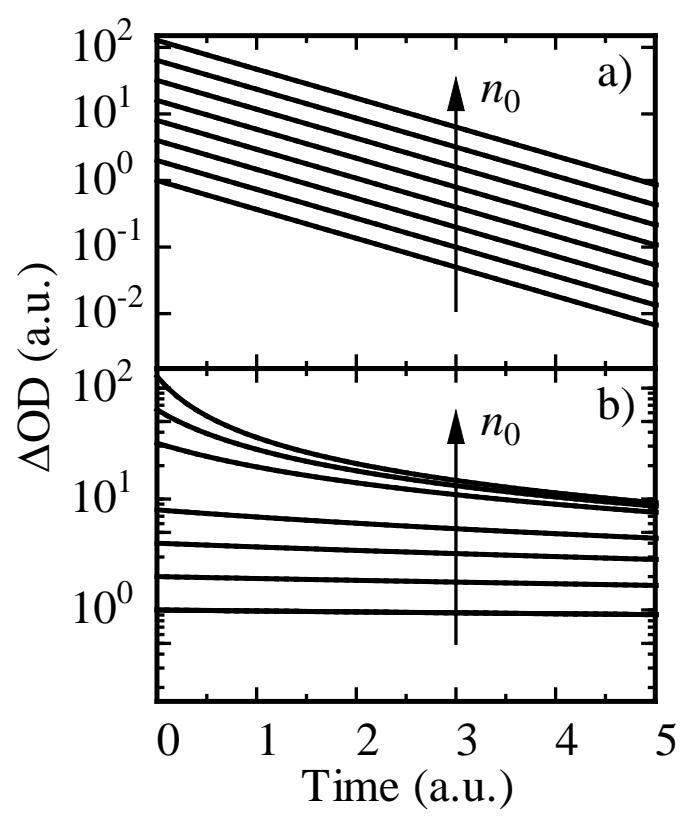

Fig. 15 


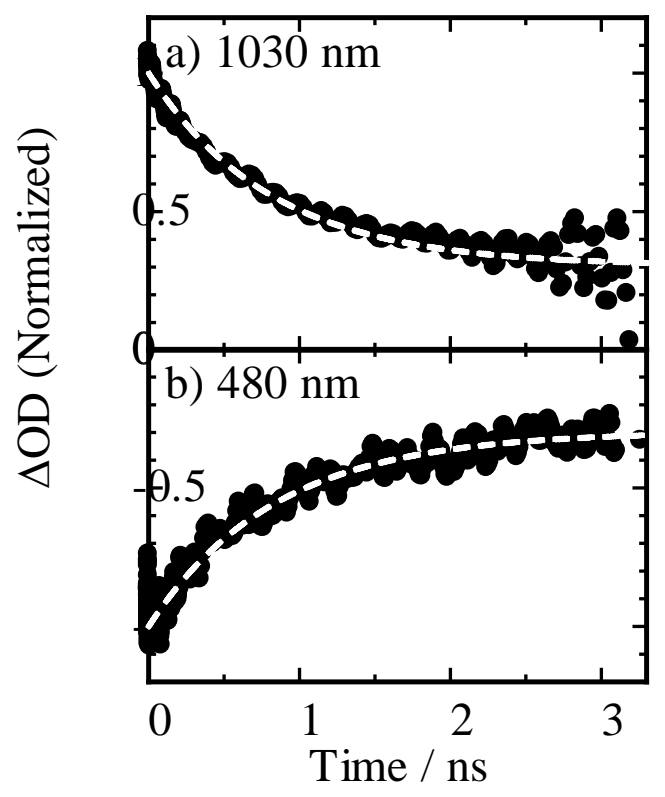

Fig. 16 


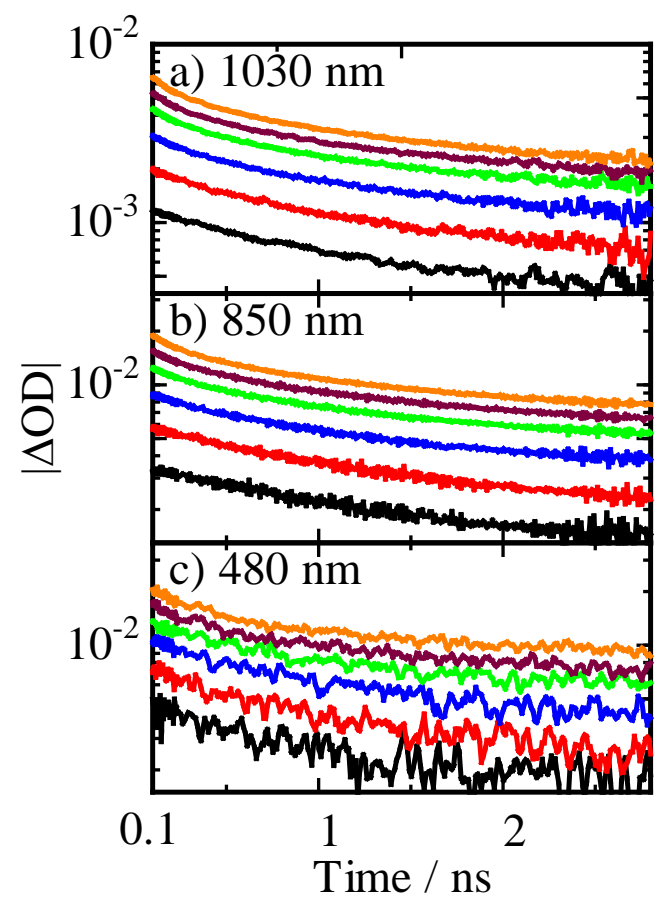

Fig. 17 


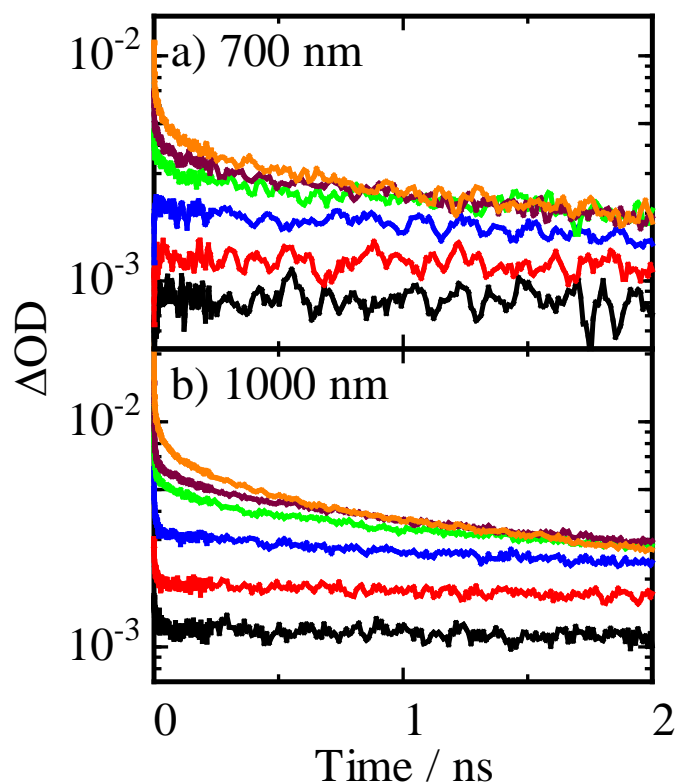

Fig. 18 


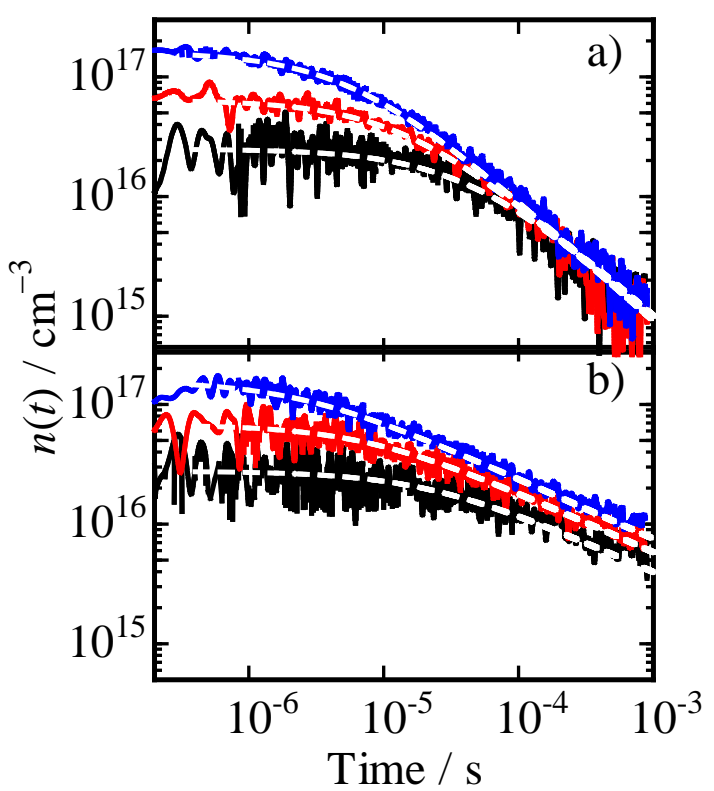

Fig. 19 

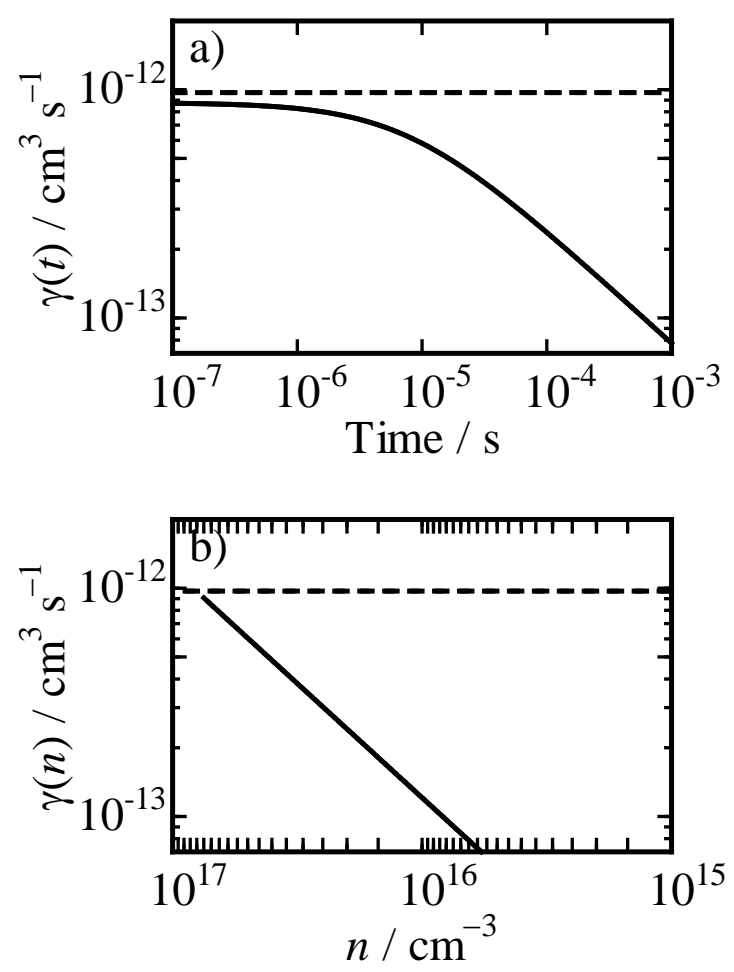

Fig. 20 


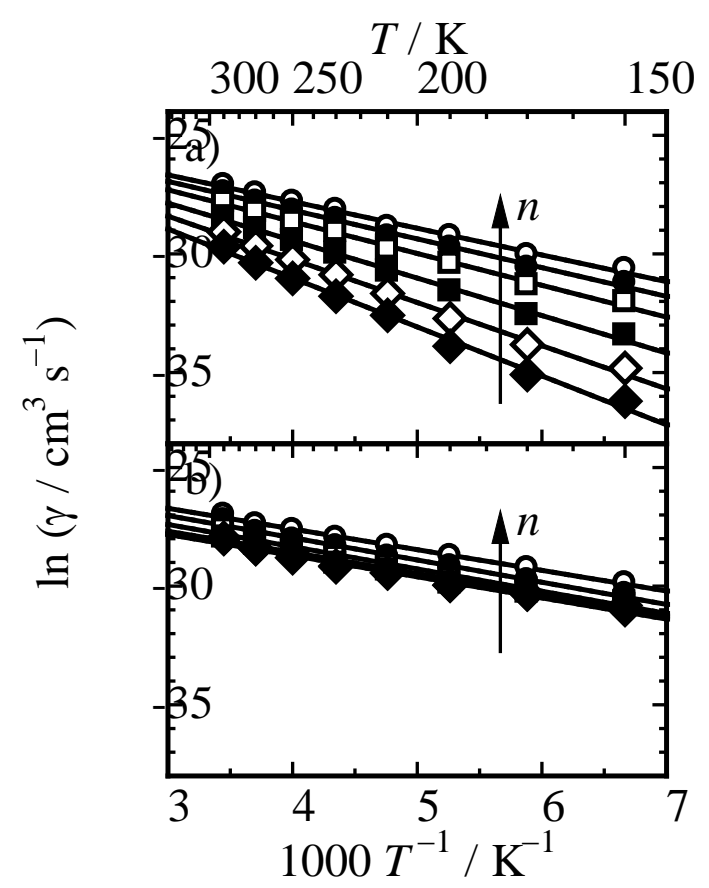

Fig. 21 
Table 1. Efficiency of each photovoltaic conversion event in P3HT:PCBM solar cells ${ }^{\mathrm{a}}[19]$

\begin{tabular}{cccccc}
\hline blend films & $\eta_{\mathrm{ED}}$ & $\eta_{\mathrm{CT}}$ & $\eta_{\mathrm{CD}}$ & $\eta_{\mathrm{CC}}$ & IQE / \% \\
\hline RRa-P3HT:PCBM & 1 & 1 & 0.31 & 0.15 & $5^{\mathrm{b}}$ \\
RR-P3HT:PCBM & & & & & \\
before annealing & 0.93 & 1 & 0.80 & $\sim 0.6-0.7$ & $42-55^{\mathrm{c}}$ \\
RR-P3HT:PCBM & & & & & \\
after annealing & 0.89 & 1 & 0.93 & $>0.9$ & $75-83^{\mathrm{d}}$ \\
\end{tabular}

${ }^{\mathrm{a}} \eta_{\mathrm{ED}}$ : Exciton diffusion efficiency to the interface of P3HT/PCBM, $\eta_{\mathrm{CT}}$ : Charge transfer efficiency at the P3HT/PCBM interface, $\eta_{\mathrm{CD}}$ : Overall charge dissociation efficiency, $\eta_{\text {Cc: }}$ Charge collection efficiency, IQE: internal quantum efficiency at $400 \mathrm{~nm}$, which is calculated by IQE $=\mathrm{EQE} / \eta_{\mathrm{A}}$ where $\mathrm{EQE}$ is the external quantum efficiency at $400 \mathrm{~nm}$ and $\eta_{\mathrm{A}}$ is estimated from twice the absorbance at $400 \mathrm{~nm}$ under the following assumptions: a) 4\% incident light loss at the air/glass interface and b) $100 \%$ reflection of the $\mathrm{Al}$ electrode.

${ }^{\mathrm{b}}$ taken from Ref 79.

${ }^{\mathrm{c}}$ taken from Refs 57 and 78.

${ }^{\mathrm{d}}$ taken from Refs 57, 78, and 80. 